\title{
Unified Approach to Spectral Properties of Multipliers
}

\author{
Mikael Lindström, Santeri Miihkinen* and David Norrbo
}

\begin{abstract}
Let $\mathbb{B}_{n}$ be the open unit ball in $\mathbb{C}^{n}$. We characterize the spectra of pointwise multipliers $M_{u}$ acting on Banach spaces of analytic functions on $\mathbb{B}_{n}$ satisfying some general conditions. These spaces include Bergman-Sobolev spaces $A_{\alpha, \beta}^{p}$, Bloch-type spaces $\mathcal{B}_{\alpha}$, weighted Hardy spaces $H_{w}^{p}$ with Muckenhoupt weights and Hardy-Sobolev Hilbert spaces $H_{\beta}^{2}$. Moreover, we describe the essential spectra of multipliers in most of the aforementioned spaces, in particular, in those spaces for which the set of multipliers is a subset of the ball algebra.
\end{abstract}

\section{Introduction and preliminaries}

In a very recent article [10], Cao, He, and Zhu considered the multiplication operator $M_{u}$ acting on the Hardy-Sobolev Hilbert space and characterized the spectrum and essential spectrum of $M_{u}$. In the present work, we extend and generalize the results obtained there from Hardy-Sobolev Hilbert space to the Bergman-Sobolev and Bloch-type spaces of the open unit ball $\mathbb{B}_{n}$ of $\mathbb{C}^{n}$ and weighted Hardy spaces of the open unit disk $\mathbb{D}$ with Muckenhoupt weights. In particular, our main focus is to allow the multiplier space $M\left(X\left(\mathbb{B}_{n}\right)\right)$ to be contained in the ball algebra, which holds for example for certain Bergman-Sobolev spaces and Bloch-type spaces. We formulate our results on spectral properties of $M_{u}$ acting on a Banach space $X\left(\mathbb{B}_{n}\right)$ of analytic functions in $\mathbb{B}_{n}$, where $X\left(\mathbb{B}_{n}\right)$ satisfies very general and natural properties regarding its multiplier space and the norm topology. Consequently, we approach the spectral properties of multipliers in a unified manner and key examples of such spaces include the aforementioned spaces. Aside from obtaining a description of the spectrum for all spaces satisfying the mentioned properties, we also have to develop some new techniques to determine the essential spectrum of $M_{u}$ regarding the non-Hilbert space case. Other previous work regarding spectral and related properties of multiplication operators on analytic function spaces includes [3, 5, 8, 9, 14, 18, 19].

The article is organised as follows. In Section 2, we introduce general Banach spaces $X\left(\mathbb{B}_{n}\right)$ of analytic functions on $\mathbb{B}_{n}$ and give central concrete examples of them. Section 3

Received December 11, 2019; Accepted February 16, 2020.

Communicated by Xiang Fang.

2010 Mathematics Subject Classification. 47B35, 47B38.

Key words and phrases. spectrum, essential spectrum, Hardy-Sobolev spaces, Bergman-Sobolev spaces, multiplication operator.

*Corresponding author. 
focuses on the spectrum of $M_{u}$ by first establishing a characterization of invertibility of $M_{u}$ and then obtaining the spectrum of $M_{u}$ and giving admissible examples of spaces on which $M_{u}$ can be defined. In Section 4, we begin with a characterization of the essential spectrum in the high-dimensional case $n>1$. Then we consider the case $n=1$ by first establishing a characterization of the Fredholmness of $M_{u}$ when conditions $(\mathrm{I}),(\mathrm{IV})$ and $M(X(\mathbb{D}))=$ $H^{\infty}(\mathbb{D})$ hold. Examples of spaces satisfying the previous conditions are also given. Next, we consider the difficult case when $M(X(\mathbb{D})) \subset A(\mathbb{D})$ (or $u \in M(X(\mathbb{D})) \cap A(\mathbb{D})$ ) and starting off with the space $X(\mathbb{D})=\mathcal{B}_{\alpha}(\mathbb{D})$ for $0<\alpha \leq 1$ and showing that the condition, earlier observed to be sufficient for the Fredholmness of $M_{u}$, is also necessary. Finally, we show the necessity of the condition in the case of those Bergman-Sobolev spaces $A_{\alpha, \beta}^{p}(\mathbb{D})$ for which $M\left(A_{\alpha, \beta}^{p}(\mathbb{D})\right) \subset A(\mathbb{D})$. From these two cases we obtain the essential spectrum of $M_{u}$ for several scales of spaces $\mathcal{B}_{\alpha}(\mathbb{D})$ and $A_{\alpha, \beta}^{p}(\mathbb{D})$ as the main result of Section 4 .

To conclude, our main result regarding the spectra of multiplication operators acting on $X\left(\mathbb{B}_{n}\right)$ is Theorem 3.2 . The essential spectra of operators $M_{u}$ acting on certain spaces $X(\mathbb{D})$ having their multiplier spaces $M(X(\mathbb{D}))$ contained in the disk algebra are described in Theorem 4.13. In the case of general $X(\mathbb{D})$ with $M(X(\mathbb{D}))=H^{\infty}(\mathbb{D})$, the essential spectra of operators $M_{u}$ are characterized in Theorem 4.5. In Theorem 4.1, we present the high-dimensional case $n>1$ concerning the essential spectra of operators $M_{u}$ acting on general spaces $X\left(\mathbb{B}_{n}\right)$.

Now we introduce some definitions and notations. Throughout this article, let $\mathbb{Z}_{\geq a}=$ $\{n \in \mathbb{Z}: n \geq a\}$ and $\mathbb{Z}_{>a}=\{n \in \mathbb{Z}: n>a\}$, where $a \in \mathbb{R}$. Furthermore, let $\mathbb{B}_{n}=$ $\left\{z \in \mathbb{C}^{n}:|z|<1\right\}, n \in \mathbb{Z}_{\geq 1}$, be the open unit ball in $\mathbb{C}^{n}$ and $\mathbb{D}=\mathbb{B}_{1}$. Moreover, let $\mathcal{H}\left(\mathbb{B}_{n}\right)$ be the space of all analytic functions $f: \mathbb{B}_{n} \rightarrow \mathbb{C}$ and $\mathcal{P}\left(\mathbb{B}_{n}\right)$ be the set of all analytic polynomials $p: \mathbb{B}_{n} \rightarrow \mathbb{C}$ such that $p(z)=\sum_{k \in J} c_{k} z^{k}$, where $J \subset \mathbb{Z}_{\geq 0}^{n}$ is a finite set, $k=\left(k_{1}, \ldots, k_{n}\right) \in \mathbb{Z}_{\geq 0}^{n},|k|=k_{1}+\cdots+k_{n}, z^{k}=z_{1}^{k_{1}} \cdots z_{n}^{k_{n}}$ and $c_{k} \in \mathbb{C}$ for $k \in J$.

We also recall that a bounded linear operator $T$ acting on a Banach space is Fredholm if it has closed range and both kernel and cokernel of $T$ are finite dimensional. The essential spectrum $\sigma_{e}(T)$ of an operator $T$ is defined as $\sigma_{e}(T)=\{\lambda \in \mathbb{C}: T-\lambda I$ is not Fredholm $\}$, where $I$ is the identity operator, and the reader may observe that $\sigma_{e}(T)$ is a subset of the spectrum $\sigma(T)$. See [1] for more details on Fredholm properties of bounded operators.

For any $f \in \mathcal{H}\left(\mathbb{B}_{n}\right)$, the gradient of $f$ is given by

$$
\nabla f(z)=\left(\frac{\partial f}{\partial z_{1}}, \ldots, \frac{\partial f}{\partial z_{n}}\right)
$$

and will be denoted $\operatorname{Df}(z)$ in the case $n=1$.

Let $\beta \in \mathbb{R}$ and $f \in \mathcal{H}\left(\mathbb{B}_{n}\right)$. The fractional radial derivative $R^{\beta}$ is given by

$$
R^{\beta} f(z)=\sum_{k=1}^{\infty} k^{\beta} f_{k}(z),
$$


where $f(z)=\sum_{k=0}^{\infty} f_{k}(z)$ is the homogeneous expansion of $f \in \mathcal{H}\left(\mathbb{B}_{n}\right)$. Let $I: \mathcal{H}\left(\mathbb{B}_{n}\right) \rightarrow$ $\mathcal{H}\left(\mathbb{B}_{n}\right)$ be the identity operator. The operator $(I+R)^{\beta}$ will also be used and is naturally defined by

$$
(I+R)^{\beta} f(z)=\sum_{k=0}^{\infty}(1+k)^{\beta} f_{k}(z) .
$$

For expressing asymptotic behaviour, the notation $a_{k} \sim b_{k}$ as $k \rightarrow \infty$ means $\lim _{k \rightarrow \infty} \frac{a_{k}}{b_{k}}$ $=1$. Moreover, by $a(x) \gtrsim b(x)$ (or $a(x) \lesssim b(x)$ ) we indicate the existence of a constant $C>0$ independent of $x$ such that $a(x) \geq C b(x)$ (or $a(x) \leq C b(x)$ ) for all $x$ in some implicit set. If both $a(x) \gtrsim b(x)$ and $a(x) \lesssim b(x)$ hold, we write $a(x) \asymp b(x)$. When two Banach spaces $X_{1}$ and $X_{2}$ are isomorphic, we use the notation $X_{1} \simeq X_{2}$.

\section{Conditions and examples}

We deal with a vector space $X\left(\mathbb{B}_{n}\right)$ of analytic functions on $\mathbb{B}_{n}$ and a norm $\|\cdot\|_{X}$ on it, that renders $X\left(\mathbb{B}_{n}\right)$ a Banach space. As usual, for each $z \in \mathbb{B}_{n}$, the evaluation functional $\delta_{z}$ is defined by $\delta_{z}(f)=f(z)$ for all $f \in X\left(\mathbb{B}_{n}\right)$. We assume that $X\left(\mathbb{B}_{n}\right)$ contains the constant functions, so then all $\delta_{z}$ are non-zero. Furthermore, we associate to $X\left(\mathbb{B}_{n}\right)$ another Banach space $Y\left(\mathbb{B}_{n}\right) \subset \mathcal{H}\left(\mathbb{B}_{n}\right)$ containing the constant functions and equipped with the norm $\|\cdot\|_{Y}$ as will be explained below.

The Banach spaces $X\left(\mathbb{B}_{n}\right)$ and $Y\left(\mathbb{B}_{n}\right)$ are often assumed to satisfy the first three conditions below:

(I) The topologies induced by $\|\cdot\|_{X}$ and $\|\cdot\|_{Y}$ are both finer than the compact-open topology $\tau_{0}$. In particular, for every $z \in \mathbb{B}_{n}, \delta_{z}$ is a bounded linear functional on both $X\left(\mathbb{B}_{n}\right)$ and $Y\left(\mathbb{B}_{n}\right)$.

Let

$$
M\left(X\left(\mathbb{B}_{n}\right)\right)=\left\{u \in \mathcal{H}\left(\mathbb{B}_{n}\right): u f \in X\left(\mathbb{B}_{n}\right) \text { for all } f \in X\left(\mathbb{B}_{n}\right)\right\} .
$$

Using condition (I) and the closed graph theorem, it follows that every $u \in M\left(X\left(\mathbb{B}_{n}\right)\right)$ induces a bounded linear operator $M_{u}: X\left(\mathbb{B}_{n}\right) \rightarrow X\left(\mathbb{B}_{n}\right)$.

(II) For some $N \in \mathbb{Z}_{\geq 1}$ it holds that $\|f\|_{X} \asymp|f(0)|+\left\|R^{N} f\right\|_{Y}$ for all $f \in \mathcal{H}\left(\mathbb{B}_{n}\right)$.

Condition (II) describes a relationship between the Banach spaces $X\left(\mathbb{B}_{n}\right)$ and $Y\left(\mathbb{B}_{n}\right)$ such that Lemma 3.1 holds. Since the lemma is trivial for spaces $X\left(\mathbb{B}_{n}\right)$ with $M\left(X\left(\mathbb{B}_{n}\right)\right)=$ $H^{\infty}\left(\mathbb{B}_{n}\right)$, this condition may be omitted when such spaces are considered. For these spaces we have $Y\left(\mathbb{B}_{n}\right)=X\left(\mathbb{B}_{n}\right)$.

(III) $H^{\infty}\left(\mathbb{B}_{n}\right) \subset M\left(Y\left(\mathbb{B}_{n}\right)\right)$.

By condition (I) it is well-known that $\sup _{z \in \mathbb{B}_{n}}|u(z)| \leq\left\|M_{u}\right\|$ for all $u \in M\left(X\left(\mathbb{B}_{n}\right)\right)$, so $M\left(X\left(\mathbb{B}_{n}\right)\right) \subset H^{\infty}\left(\mathbb{B}_{n}\right)$ and $M\left(Y\left(\mathbb{B}_{n}\right)\right)=H^{\infty}\left(\mathbb{B}_{n}\right)$, where also condition (III) is used in the second statement. Since $u \mapsto M_{u}$ is bounded according to the bounded inverse 
theorem, it follows from the boundedness of $M_{u}$, that there exists a constant $C>0$ such that $\left\|M_{u} g\right\|_{Y} \leq C\|u\|_{\infty}\|g\|_{Y}$ for all $g \in Y\left(\mathbb{B}_{n}\right)$ and $u \in M\left(Y\left(\mathbb{B}_{n}\right)\right)$.

When considering the case $n=1$, we will need the following condition to determine the essential spectra of the multiplication operator generated by $u \in M(X(\mathbb{D}))$.

$(\mathrm{IV})$ If $f \in X(\mathbb{D})$ has a zero at $z_{0} \in \mathbb{D}$, then $\frac{f(z)}{z-z_{0}} \in X(\mathbb{D})$.

Lemma 2.1. Let $f \in \mathcal{H}(\mathbb{D})$ and $v: \mathbb{D} \rightarrow[0, \infty)$ be a bounded function such that $v(z)=$ $v(|z|)$ for all $z \in \mathbb{D}$. Moreover, let $N \in \mathbb{Z}_{\geq 0}$ be such that

$$
\sup _{z \in \mathbb{D}} v(z)\left|D^{N} f(z)\right|<\infty
$$

If $z_{0} \in \mathbb{D}$ is a zero of $f$, then

$$
\sup _{z \in \mathbb{D}} v(z)\left|D^{N} \frac{f(z)}{z-z_{0}}\right|<\infty
$$

Proof. Let $g(z)=\frac{f(z)}{z-z_{0}}$. Since $f$ is analytic with a zero at $z_{0}$ we have $D^{N} g \in \mathcal{H}(\mathbb{D})$. Thus, $h(z)=v(z)\left|D^{N} g(z)\right|$ is bounded on $\mathbb{D}$ if and only if $h$ is bounded near the boundary. For $z \in T=\left\{z \in \mathbb{D}:|z|>\frac{1+\left|z_{0}\right|}{2}\right\}$, we have the following estimate

$$
\left|D^{N} g(z)\right|=\left|\sum_{j=0}^{N}\left(\begin{array}{c}
N \\
j
\end{array}\right) D^{j} f(z) D^{N-j}\left(z-z_{0}\right)^{-1}\right| \leq \sum_{j=0}^{N} \frac{N !\left|D^{j} f(z)\right|}{\left(|z|-\left|z_{0}\right|\right)^{N-j+1}}
$$

Furthermore, for $k \geq 0$ we have

$$
\begin{aligned}
\left|D^{k} f(z)\right| & \leq\left|\int_{C_{z}} D^{k+1} f(w) d w\right|+\left|D^{k} f(0)\right| \\
& \leq|z| \sup _{|w|=|z|}\left|D^{k+1} f(w)\right|+\left|D^{k} f(0)\right| \\
& \leq \sup _{|w|=|z|}\left|D^{k+1} f(w)\right|+\left|D^{k} f(0)\right|,
\end{aligned}
$$

where $C_{z}$ is the line from 0 to $z$ in $\mathbb{D}$.

By induction, it can be shown that

$$
\left|D^{k} f(z)\right| \leq \sup _{|y|=|z|}\left|D^{N} f(y)\right|+\sum_{j=0}^{N-k-1}\left|D^{k+j} f(0)\right|
$$

for $0 \leq k \leq N$. Moreover, from the fact that $\sup _{z \in \mathbb{D}} \sup _{|w|=|z|}$ is interchangable with 
$\sup _{w \in \mathbb{D}}$ and $v(z)=v(|z|)$ we now obtain

$$
\begin{aligned}
v(z)\left|D^{k} f(z)\right| & \leq \sup _{|y|=|z|} v(z)\left|D^{N} f(y)\right|+v(z) \sum_{j=0}^{N-k-1}\left|D^{k+j} f(0)\right| \\
& =\sup _{|y|=|z|} v(y)\left|D^{N} f(y)\right|+v(z) \sum_{j=0}^{N-k-1}\left|D^{k+j} f(0)\right| \\
& \leq \sup _{z \in \mathbb{D}} v(z)\left|D^{N} f(z)\right|+\sup _{z \in \mathbb{D}} v(z) \sum_{j=0}^{N-1}\left|D^{j} f(0)\right|=M_{f, N, v}<\infty
\end{aligned}
$$

for all $z \in \mathbb{D}$. Especially for $z \in T$, using (2.1), we have

$$
v(z)\left|D^{N} g(z)\right| \leq \sum_{k=0}^{N} \frac{N ! v(z)\left|D^{k} f(z)\right|}{\left(|z|-\left|z_{0}\right|\right)^{N-k+1}} \leq M_{f, N, v} \sum_{k=0}^{N} \frac{N ! 2^{N-k+1}}{\left(1-\left|z_{0}\right|\right)^{N-k+1}}<\infty,
$$

which proves the lemma.

Next, we list a number of spaces satisfying the above conditions (I)-(IV). However, in Example 2.4 we consider spaces $X\left(\mathbb{B}_{n}\right)$ for which $M\left(X\left(\mathbb{B}_{n}\right)\right)=H^{\infty}\left(\mathbb{B}_{n}\right)$, implying that condition (II) is irrelevant.

Example 2.2. For $\alpha>0$ the Bloch-type space $X\left(\mathbb{B}_{n}\right)=\mathcal{B}_{\alpha}\left(\mathbb{B}_{n}\right)$ is the space of all $f \in \mathcal{H}\left(\mathbb{B}_{n}\right)$ satisfying $\|f\|_{\mathcal{B}_{\alpha}}=|f(0)|+\sup _{z \in \mathbb{B}_{n}}\left(1-|z|^{2}\right)^{\alpha}|\nabla f(z)|<\infty$, see [21]. To these spaces correspond

$$
Y\left(\mathbb{B}_{n}\right)=H_{\alpha}^{\infty}\left(\mathbb{B}_{n}\right)=\left\{f \in \mathcal{H}\left(\mathbb{B}_{n}\right):\|f\|_{H_{\alpha}^{\infty}}=\sup _{z \in \mathbb{B}_{n}}\left(1-|z|^{2}\right)^{\alpha}|f(z)|<\infty\right\},
$$

see $[6]$. The little Bloch-type space $\mathcal{B}_{0, \alpha}\left(\mathbb{B}_{n}\right)$ is the subspace of $\mathcal{B}_{\alpha}\left(\mathbb{B}_{n}\right)$ satisfying $\lim _{|z| \rightarrow 1}$ $\left(1-|z|^{2}\right)^{\alpha}|\nabla f(z)|=0$. It is well-known that these spaces obey (I). Let $\|f\|_{\mathcal{B} R_{\alpha}}=|f(0)|+$ $\sup _{z \in \mathbb{B}_{n}}\left(1-|z|^{2}\right)^{\alpha}|R f(z)|$. According to Theorem 7.1 in [21], it holds that

$$
\left\{f \in \mathcal{H}\left(\mathbb{B}_{n}\right):\|f\|_{\mathcal{B}_{\alpha}}<\infty\right\}=\left\{f \in \mathcal{H}\left(\mathbb{B}_{n}\right):\|f\|_{\mathcal{B} R_{\alpha}}<\infty\right\}
$$

Therefore it follows from the bounded inverse theorem that $\|\cdot\|_{\mathcal{B}_{\alpha}} \asymp\|\cdot\|_{\mathcal{B} R_{\alpha}}$ and, hence, both of these spaces satisfy condition (II). Condition (III) holds by definition. We will consider the space $\left(\mathcal{B}_{\alpha}(\mathbb{D}),\|\cdot\|_{\mathcal{B}_{\alpha}}\right)$ in the one-dimensional case.

By Theorem 2.1(i) in [17], for $0<\alpha<1, u \in M\left(\mathcal{B}_{\alpha}(\mathbb{D})\right)$ if and only if $u \in \mathcal{B}_{\alpha}(\mathbb{D}) \cap$ $H^{\infty}(\mathbb{D})=\mathcal{B}_{\alpha}(\mathbb{D}) \subset A(\mathbb{D})$, where the inclusion is found in Theorem 7.9 in 21 . When $\alpha=1$, we get from Theorem 2.1(ii) in 17 that $u \in M\left(\mathcal{B}_{\alpha}(\mathbb{D})\right)$ if and only if

$$
\sup _{z \in \mathbb{D}}\left|u^{\prime}(z)\right|\left(1-|z|^{2}\right) \log \left(\frac{e}{1-|z|^{2}}\right)<\infty \quad \text { and } \quad u \in H^{\infty}(\mathbb{D}) .
$$


Therefore $u \in \mathcal{B}_{0,1}(\mathbb{D})$. Finally by Theorem 2.1(iii) in $[17$ we have for $\alpha>1$ that $u \in M\left(\mathcal{B}_{\alpha}(\mathbb{D})\right)$ if and only if $u \in \mathcal{B}_{1}(\mathbb{D}) \cap H^{\infty}(\mathbb{D})=H^{\infty}(\mathbb{D})$. According to Lemma 2.1 a function belonging to $\mathcal{B}_{\alpha}(\mathbb{D})$ will remain in $\mathcal{B}_{\alpha}(\mathbb{D})$ after removing a finite number of zeros $z_{0}$ through division by $z-z_{0}$, which proves $(\mathrm{IV})$. The notations $\mathcal{B}(\mathbb{D})$ and $\mathcal{B}_{0}(\mathbb{D})$ stand for $\mathcal{B}_{1}(\mathbb{D})$ and $\mathcal{B}_{0,1}(\mathbb{D})$ respectively.

Example 2.3. Let $\beta \geq 0, \alpha \geq-1$ and $1 \leq p<\infty$. The holomorphic Sobolev space $A_{\alpha, \beta}^{p}\left(\mathbb{B}_{n}\right)$ is defined by

$$
A_{\alpha, \beta}^{p}\left(\mathbb{B}_{n}\right)=\left\{f \in \mathcal{H}\left(\mathbb{B}_{n}\right):\|f\|_{A_{\alpha, \beta}^{p}}<\infty\right\},
$$

where the norm is defined by

$$
\|f\|_{A_{\alpha, \beta}^{p}}=\left\|(I+R)^{\beta} f\right\|_{A_{\alpha}^{p}}=\left(\int_{\mathbb{B}_{n}}\left|(I+R)^{\beta} f(z)\right|^{p} d A_{\alpha}(z)\right)^{1 / p}
$$

for $\alpha>-1$ and

$$
\|f\|_{A_{-1, \beta}^{p}}=\left\|(I+R)^{\beta} f\right\|_{H^{p}}=\left(\int_{\partial \mathbb{B}_{n}}\left|(I+R)^{\beta} f(z)\right|^{p} d S(z)\right)^{1 / p} .
$$

Furthermore, $d A_{\alpha}(z)=\frac{\Gamma(n+\alpha+1)}{n ! \Gamma(\alpha+1)}\left(1-|z|^{2}\right)^{\alpha} d A(z)$, where $d A(z)$ is the $2 n$-dimensional Lebesgue measure normalized so that $\int_{\mathbb{B}_{n}} d A(z)=1$, and hence, $\int_{\mathbb{B}_{n}} d A_{\alpha}(z)=1$ for every $\alpha>-1$. The notation $d S(z)$ stands for the surface measure satisfying $\int_{\partial \mathbb{B}_{n}} d S(z)=1$. The holomorphic Sobolev spaces can be partitioned into the Bergman-Sobolev spaces, $\alpha>-1$, and the Hardy-Sobolev spaces, $H_{\beta}^{p}\left(\mathbb{B}_{n}\right)=A_{-1, \beta}^{p}\left(\mathbb{B}_{n}\right)$. In case of $\beta=0$, these spaces are called the weighted Bergman spaces $A_{\alpha}^{p}\left(\mathbb{B}_{n}\right)=A_{\alpha, 0}^{p}\left(\mathbb{B}_{n}\right)$ with $\alpha>-1$ and the Hardy spaces $H^{p}\left(\mathbb{B}_{n}\right)=A_{-1,0}^{p}\left(\mathbb{B}_{n}\right)$.

For $p \geq 1, \alpha_{j}>-1, \beta_{j} \geq 0(j=1,2)$, with $\alpha_{1}-\alpha_{2}=p\left(\beta_{1}-\beta_{2}\right)$, the following equivalence holds by Theorem 5.12 in [4] (see also [11]):

$$
A_{\alpha_{1}, \beta_{1}}^{p}\left(\mathbb{B}_{n}\right) \simeq A_{\alpha_{2}, \beta_{2}}^{p}\left(\mathbb{B}_{n}\right)
$$

where the isomorphism is given by the identity operator, and hence, the spaces have equivalent norms. By the same theorem, one also obtains the statement 2.2 for $\alpha_{1}=-1$ and $p=2$. From this it follows that for $\beta_{1}<\frac{1+\alpha_{1}}{p}$, where equality may be used in the case of $p=2$, we have $A_{\alpha_{1}, \beta_{1}}^{p}\left(\mathbb{B}_{n}\right) \simeq A_{\alpha_{1}-\beta_{1} p, 0}^{p}\left(\mathbb{B}_{n}\right)$. The right-hand side is a weighted Bergman space or $H^{2}\left(\mathbb{B}_{n}\right)$, hence, $M\left(A_{\alpha, \beta}^{p}\left(\mathbb{B}_{n}\right)\right)=H^{\infty}\left(\mathbb{B}_{n}\right)$ for $\beta<\frac{1+\alpha}{p}$, where equality may be used in the case of $p=2$. Regarding the case $n=1$, if $\beta>\frac{2+\alpha}{p}$, then $A_{\alpha, \beta}^{p}(\mathbb{D})$ is an algebra and $M\left(A_{\alpha, \beta}^{p}(\mathbb{D})\right)=A_{\alpha, \beta}^{p}(\mathbb{D})$, see $[5$. In this setting, there is a $b<\beta$ satisfying $0<b-\frac{2+\alpha}{p}<1$, so that

$$
A_{\alpha, \beta}^{p}(\mathbb{D}) \subset A_{\alpha, b}^{p}(\mathbb{D}) \subset \Lambda_{b-\frac{2+\alpha}{p}}(\mathbb{D}) \subset A(\mathbb{D}),
$$


where $\Lambda_{b-\frac{2+\alpha}{p}}$ is a Lipschitz space, see [21]. The first inclusion follows from 2.2 , the second inclusion can be found in Theorem 5.5 in [4] and the last one is given by Theorem 7.9 in [21. Furthermore, by Proposition 2.2 in [11, we have for $p \geq 1, \alpha \geq-1$, and every positive integer $N$ that

$$
\|f\|_{A_{\alpha, N}^{p}} \asymp \sum_{j=0}^{N-1}\left|D^{j} f(0)\right|+\left\|D^{N} f\right\|_{A_{\alpha}^{p}}
$$

for $f \in \mathcal{H}(\mathbb{D})$. Next, we check the conditions (I)-(IV).

The topology generated by $\|\cdot\|_{A_{\alpha, \beta}^{p}}$ is finer than the compact-open topology $\tau_{0}$, so condition (I) holds. Indeed, the statement follows from Lemma 5.6 in 4 with the use of supremum over an arbitrary compact subset of $\mathbb{B}_{n}$. Hereafter, we will assume that $\beta \geq \frac{1+\alpha}{p}$. For smaller $\beta$ it was mentioned that the multiplier space is $H^{\infty}\left(\mathbb{B}_{n}\right)$ which is considered in Example 2.4, where the the space $A_{\alpha, \beta}^{p}\left(\mathbb{B}_{n}\right)$ can be viewed as a weighted Bergman space.

In the case $N>\beta-\frac{\alpha+1}{p} \geq 0$, an application of 2.2 gives that $f \in A_{\alpha, \beta}^{p}\left(\mathbb{B}_{n}\right)$ if and only if $f \in A_{(N-\beta) p+\alpha, N}^{p}\left(\mathbb{B}_{n}\right)$ if and only if $R^{N} f \in A_{(N-\beta) p+\alpha}^{p}\left(\mathbb{B}_{n}\right)$.

Therefore, let $X\left(\mathbb{B}_{n}\right)=A_{\alpha, \beta}^{p}\left(\mathbb{B}_{n}\right)$ and $Y\left(\mathbb{B}_{n}\right)=A_{(N-\beta) p+\alpha}^{p}\left(\mathbb{B}_{n}\right)$, where $N=\inf \{\widehat{N} \in$ $\left.\mathbb{Z}_{\geq 1}: \widehat{N}>\beta-\frac{\alpha+1 / 2}{p}\right\}$. Moreover, for $f \in \mathcal{H}\left(\mathbb{B}_{n}\right)$ we have

$$
\left\|(I+R)^{\beta} f\right\|_{A_{\alpha}^{p}} \asymp|f(0)|+\left\|R^{\beta} f\right\|_{A_{\alpha}^{p}},
$$

according to Lemma 2.5. Condition (II) follows by first using the equivalence of the norms $\|\cdot\|_{A_{\alpha, \beta}^{p}}$ and $\|\cdot\|_{A_{(N-\beta) p+\alpha, N}^{p}}$ by 2.2), and then applying (2.4) to the latter norm. Furthermore, it holds that $M\left(A_{\alpha}^{p}\left(\mathbb{B}_{n}\right)\right)=H^{\infty}\left(\mathbb{B}_{n}\right)$, which shows that condition (III) is satisfied.

Let us check the condition $(\mathrm{IV})$ for $A_{\alpha, \beta}^{p}(\mathbb{D})$. We assume that $f \in A_{\alpha, \beta}^{p}(\mathbb{D})$ has a zero at $z=z_{0}$. Let us show that $\frac{f}{z-z_{0}} \in A_{\alpha, \beta}^{p}(\mathbb{D})$ by establishing that $R^{N}\left(\frac{f}{z-z_{0}}\right) \in A_{(N-\beta) p+\alpha}^{p}(\mathbb{D})$. Let us take $\left|z_{0}\right|<r<1$. We may assume that $|z| \geq r$, since $R^{N}\left(\frac{f}{z-z_{0}}\right) \in H(\mathbb{D})$ is bounded on $r \mathbb{D}$. We will utilize the following formula given in Proposition 6 in [10]:

$$
R^{N}\left(\frac{f(z)}{z-z_{0}}\right)=\frac{(-1)^{N}}{\left(z-z_{0}\right)^{N+1}} \sum_{k=0}^{N}(-1)^{k}\left(\begin{array}{c}
N+1 \\
k
\end{array}\right)\left(z-z_{0}\right)^{k} R^{N}\left(\left(z-z_{0}\right)^{N-k} f\right),
$$

where $r \leq|z|<1$. It suffices to show that $R^{N}\left(\left(z-z_{0}\right) f\right) \in A_{(N-\beta) p+\alpha}^{p}(\mathbb{D})$, which implies that $R^{N}\left(\left(z-z_{0}\right)^{N-k} f\right) \in A_{(N-\beta) p+\alpha}^{p}(\mathbb{D})$ for $k=0,1, \ldots, N$. Using the general Leibniz rule we obtain

$$
R^{N}\left(\left(z-z_{0}\right) f\right)=\sum_{k=0}^{N}\left(\begin{array}{c}
N \\
k
\end{array}\right) R^{N-k}\left(z-z_{0}\right) R^{k}(f)=\sum_{k=0}^{N}\left(\begin{array}{l}
N \\
k
\end{array}\right) z R^{k}(f) .
$$


We observe that $\left\|z R^{k} f\right\|_{A_{(N-\beta) p+\alpha}^{p}} \leq\left\|R^{k} f\right\|_{A_{(N-\beta) p+\alpha}^{p}}$ and $R^{k} f \in A_{(N-\beta) p+\alpha}^{p}(\mathbb{D})$ if and only if

$$
\left(1-|z|^{2}\right)^{N-k} R^{(N-k)} R^{k} f=\left(1-|z|^{2}\right)^{N-k} R^{N} f \in A_{(N-\beta) p+\alpha}^{p}(\mathbb{D}),
$$

see [21, p. 75]. The last statement holds, since

$$
\left\|\left(1-|z|^{2}\right)^{N-k} R^{N} f\right\|_{A_{(N-\beta) p+\alpha}^{p}} \leq\left\|R^{N} f\right\|_{A_{(N-\beta) p+\alpha}^{p}}<\infty
$$

where we used the fact $R^{N} f \in A_{(N-\beta) p+\alpha}^{p}(\mathbb{D})$. So we have that $R^{k} f \in A_{(N-\beta) p+\alpha}^{p}(\mathbb{D})$ for $k=0,1, \ldots, N$ and consequently $R^{N}\left(\left(z-z_{0}\right) f\right) \in A_{(N-\beta) p+\alpha}^{p}(\mathbb{D})$. Therefore $R^{N}\left(\frac{f}{z-z_{0}}\right) \in$ $A_{(N-\beta) p+\alpha}^{p}(\mathbb{D})$.

It should also be mentioned that the spaces $A_{\alpha, \beta}^{p}\left(\mathbb{B}_{n}\right)$ are reflexive for $p>1$, see Proposition 5.7(iv) in 4].

Example 2.4. We consider all spaces $X\left(\mathbb{B}_{n}\right)$ that satisfies $(\mathrm{I})$ and $M\left(X\left(\mathbb{B}_{n}\right)\right)=H^{\infty}\left(\mathbb{B}_{n}\right)$. Furthermore, condition (IV) is also assumed to hold if $n=1$. Letting $Y\left(\mathbb{B}_{n}\right)=X\left(\mathbb{B}_{n}\right)$ condition (III) is also satisfied and condition (II) is irrelevant, see the remark after condition (II). These spaces include growth spaces $H_{\alpha}^{\infty}(\mathbb{D}), \alpha>0$, and weighted Hardy spaces $H_{w}^{p}(\mathbb{D}), p>1$, where $w \in\left(A^{p}\right)$, that is, $w$ satisfies the Muckenhoupt $\left(A^{p}\right)$-condition, see details in [7]. Considering the weighted Hardy spaces, condition (I) follows from the proof of Lemma 2.1 in [7. Notice that if $w \in\left(A^{p}\right), p>1$, then the critical exponent $q_{w}<p$. For $f \in \mathcal{H}(\mathbb{D})$ we have $\|f\|_{H_{w}^{p}}<\infty$ if and only if

$$
\lim _{r \rightarrow 1^{-}} \int_{-\pi}^{\pi}\left|f\left(r e^{i \theta}\right)\right|^{p} w(\theta) d \theta<\infty
$$

Since, for every $z_{0} \in \mathbb{D}$, there exists $r<1$ such that $\frac{1}{z-z_{0}}$ is bounded on $\mathbb{D} \backslash r \mathbb{D}$, condition (IV) follows. Condition (IV) is proved by similar arguments for many spaces, for example, weighted Bergman spaces, growth spaces and Hardy spaces.

Lemma 2.5. Let $\beta \geq 0$. If either $\alpha>-1$ and $p \geq 1$, or $\alpha=-1$ and $p=2$, it holds that

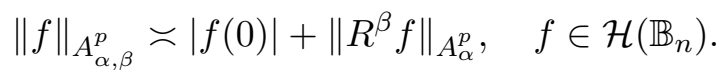

Moreover, the space $A_{\alpha, \beta}^{p}\left(\mathbb{B}_{n}\right)$ endowed with the norm defined as $\|f\|_{p, \alpha, \beta}=|f(0)|+$ $\left\|R^{\beta} f\right\|_{A_{\alpha}^{p}}$ is a Banach space.

Proof. Let $N$ be the smallest integer in the set $\mathbb{Z}_{>\beta+1}$ and $\gamma=p(N-\beta)+\alpha>-1$. By (2.2) and Lemma 1 in [10], we have $\|f\|_{A_{\alpha, \beta}^{p}} \asymp\|f\|_{A_{\gamma, N}^{p}}$ and $\|f\|_{p, \alpha, \beta} \asymp\|f\|_{p, \gamma, N}$ respectively. The norm equivalences are also well known to experts in the case $\alpha=-1$ and $p=2$. Therefore

$$
\left(A_{\alpha, \beta}^{p}\left(\mathbb{B}_{n}\right),\|\cdot\|_{p, \alpha, \beta}\right)
$$


is a Banach space, since this is true for

$$
\left(A_{\alpha, \beta}^{p}\left(\mathbb{B}_{n}\right),\|\cdot\|_{p, \gamma^{\prime}, N}\right)
$$

for all $\gamma^{\prime}>-1$, see $[20]$. It now suffices to show that $\|f\|_{A_{\gamma, N}^{p}} \asymp\|f\|_{p, \gamma, N}$. Using Jensen's inequality we have

$$
\begin{aligned}
\left|(I+R)^{N} f(z)\right| & =\left|\sum_{j=0}^{N}\left(\begin{array}{c}
N \\
j
\end{array}\right) R^{j} f(z)\right| \leq 2^{N} \sum_{j=0}^{N} \frac{1}{2^{N}}\left(\begin{array}{c}
N \\
j
\end{array}\right)\left|R^{j} f(z)\right| \\
& \leq 2^{N}\left(\sum_{j=0}^{N} \frac{1}{2^{N}}\left(\begin{array}{c}
N \\
j
\end{array}\right)\left|R^{j} f(z)\right|^{p}\right)^{1 / p} .
\end{aligned}
$$

Furthermore,

$$
\begin{aligned}
\|f\|_{A_{\gamma, N}^{p}} & =\left(\int_{\mathbb{B}_{n}}\left|(I+R)^{N} f(z)\right|^{p} d A_{\gamma}(z)\right)^{1 / p} \\
& \leq\left(2^{N(p-1)} \sum_{j=0}^{N}\left(\begin{array}{c}
N \\
j
\end{array}\right) \int_{\mathbb{B}_{n}}\left|R^{j} f(z)\right|^{p} d A_{\gamma}(z)\right)^{1 / p} \\
& \asymp\left(\sum_{j=0}^{N} \int_{\mathbb{B}_{n}}\left|R^{N} f(z)\right|^{p} d A_{p(N-j)+\gamma}(z)\right)^{1 / p} \\
& \lesssim\left(\int_{\mathbb{B}_{n}}\left|R^{N} f(z)\right|^{p} d A_{\gamma}(z)\right)^{1 / p}+|f(0)|=\|f\|_{p, \gamma, N} .
\end{aligned}
$$

Our approach to prove the converse is very similar. It holds that

$$
\begin{aligned}
\int_{\mathbb{B}_{n}}\left|R^{N} f(z)\right|^{p} d A_{\gamma}(z) & =\int_{\mathbb{B}_{n}}\left|(I+R-I)^{N} f(z)\right|^{p} d A_{\gamma}(z) \\
& \leq 2^{N(p-1)} \sum_{j=0}^{N}\left(\begin{array}{c}
N \\
j
\end{array}\right) \int_{\mathbb{B}_{n}}\left|(I+R)^{j} f(z)\right|^{p} d A_{\gamma}(z) \\
& \asymp \sum_{j=0}^{N} \int_{\mathbb{B}_{n}}\left|(I+R)^{N} f(z)\right|^{p} d A_{p(N-j)+\gamma}(z) \\
& \lesssim \int_{\mathbb{B}_{n}}\left|(I+R)^{N} f(z)\right|^{p} d A_{\gamma}(z) .
\end{aligned}
$$

From this and Lemma 5.6 in 4, it follows that there exists a constant $M=M(n, N, p, \gamma)>$ 0 such that

$$
\left\|R^{N} f\right\|_{A_{\gamma}^{p}}+|f(0)| \leq M\left\|(I+R)^{N} f\right\|_{A_{\gamma}^{p}}+|f(0)| \leq 2 M\left\|(I+R)^{N} f\right\|_{A_{\gamma}^{p}},
$$

which finishes the proof. 
3. The spectrum of $M_{u}$

Next, we will characterize the spectra of multiplication operators acting on $X\left(\mathbb{B}_{n}\right)$ in the case that there exists a space $Y\left(\mathbb{B}_{n}\right)$ such that conditions (I)-(III) are satisfied. Condition (II) is crucial in the following lemma. The corresponding results for the Hardy-Sobolev Hilbert spaces were obtained in 10 .

Lemma 3.1. Assume that (I)-(III) are satisfied and let $u \in M\left(X\left(\mathbb{B}_{n}\right)\right)$. The following statements are equivalent:

(a) $1 / u \in M\left(X\left(\mathbb{B}_{n}\right)\right)$,

(b) $1 / u \in H^{\infty}\left(\mathbb{B}_{n}\right)$,

(c) $M_{u}$ is invertible.

Proof. Assuming $1 / u \in M\left(X\left(\mathbb{B}_{n}\right)\right)$ we obtain immediately, by the remark after condition (III), that $1 / u \in H^{\infty}\left(\mathbb{B}_{n}\right)$. To prove the converse implication we will use the formula

$$
R^{N}\left(\frac{f}{u}\right)=\frac{(-1)^{N}}{u^{N+1}} \sum_{k=0}^{N}(-1)^{k}\left(\begin{array}{c}
N+1 \\
k
\end{array}\right) u^{k} R^{N}\left(u^{N-k} f\right), \quad f \in \mathcal{H}\left(\mathbb{B}_{n}\right),
$$

which can be found in Corollary 5 in [10]. The proof of the formula uses the derivative $D$, but the formula remains valid for all linear operators $S$ that admit the law $S(f g)=$ $f S g+g S f, f, g \in \mathcal{H}(\mathbb{D})$, and for which the formula is valid for $N=1$. Moreover, the dimension $n$ is irrelevant for the proof, and therefore we may replace $D$ with $R$ and also consider the formula in higher dimensions. Notice that (3.1) is invalid for $N=0$.

If $1 / u \in H^{\infty}\left(\mathbb{B}_{n}\right)$, then $u$ is uniformly bounded from below, that is, there exists a $0<c<1$ such that $\inf _{z \in \mathbb{B}_{n}}|u(z)| \geq c$ and hence formula (3.1) is applicable. By condition (II) we have that $f \in X\left(\mathbb{B}_{n}\right)$ if and only if $R^{N} f \in Y\left(\mathbb{B}_{n}\right)$.

One should also notice that $u^{k} \in M\left(X\left(\mathbb{B}_{n}\right)\right)$ and $(1 / u)^{k} \in H^{\infty}\left(\mathbb{B}_{n}\right)$ for all $k \in \mathbb{Z}_{\geq 0}$. For $f \in X\left(\mathbb{B}_{n}\right)$ we obtain that $u^{k} f \in X\left(\mathbb{B}_{n}\right)$ for all $k \in \mathbb{Z}_{\geq 0}$, and therefore,

$$
\begin{aligned}
\left\|\frac{f}{u}\right\|_{X} & \asymp\left\|R^{N}\left(\frac{f}{u}\right)\right\|_{Y}+\left|\frac{f(0)}{u(0)}\right| \\
& \leq \sum_{k=0}^{N}\left(\begin{array}{c}
N+1 \\
k
\end{array}\right) \|\left.\left(\frac{1}{u}\right)^{N+1-k} R^{N}\left(u^{N-k} f\right)\right|_{Y}+\left|\frac{f(0)}{u(0)}\right| \\
& \lesssim \sum_{k=0}^{N}\left\|\left(\frac{1}{u}\right)^{N+1-k}\right\|\left\|R_{\infty}^{N}\left(u^{N-k} f\right)\right\|_{Y}+\left|\frac{f(0)}{u(0)}\right| \\
& \leq\left(\frac{1}{c}\right)^{N+1} \sum_{k=0}^{N}\left\|R^{N}\left(u^{N-k} f\right)\right\|_{Y}+\left|\frac{f(0)}{u(0)}\right| \lesssim \sum_{k=0}^{N}\left\|u^{N-k} f\right\|_{X}+\left|\frac{f(0)}{u(0)}\right|<\infty,
\end{aligned}
$$


where the remark after condition (III) gives the second inequality. Hence, we have shown that the two statements (a) and (b) are equivalent. If $1 / u \in M\left(X\left(\mathbb{B}_{n}\right)\right)$, then clearly $f \mapsto \frac{f}{u}$ is the inverse of $M_{u}$. Conversely, if $M_{u}$ is invertible, then $M_{1 / u}$ must be the unique bounded inverse, so $1 / u \in M\left(X\left(\mathbb{B}_{n}\right)\right)$.

Theorem 3.2. Assume that (I)-(III) are satisfied and let $M_{u}: X\left(\mathbb{B}_{n}\right) \rightarrow X\left(\mathbb{B}_{n}\right)$ be a multiplication operator generated by $u \in M\left(X\left(\mathbb{B}_{n}\right)\right)$. The spectrum of $M_{u}$ is given by $\sigma\left(M_{u}\right)=\overline{u\left(\mathbb{B}_{n}\right)}$.

Proof. Let $\lambda \in \mathbb{C}$. Clearly $u-\lambda \in M\left(X\left(\mathbb{B}_{n}\right)\right)$. If $\lambda \in \overline{u\left(\mathbb{B}_{n}\right)}$, then $|u(z)-\lambda|$ is not bounded from below so $M_{u}-\lambda I=M_{u-\lambda}$ is not invertible by Lemma 3.1. Using again Lemma 3.1 . it follows that for any $\lambda \in \mathbb{C} \backslash \overline{u\left(\mathbb{B}_{n}\right)}$ the operator $M_{u}-\lambda I$ is invertible since $|u(z)-\lambda|$, in this case, is bounded from below. Hence, the spectrum is given by $\sigma\left(M_{u}\right)=\overline{u\left(\mathbb{B}_{n}\right)}$.

Remark 3.3. The above result implies that $r\left(M_{u}\right)=\|u\|_{\infty} \leq\left\|M_{u}\right\|$. Moreover, since the spectrum $\sigma\left(M_{u}\right)=\overline{u\left(\mathbb{B}_{n}\right)}$ is connected, when $u$ is continuous, any nonzero spectral radius would imply an uncountable number of points in the spectrum, from which it follows that the operator is not compact. Consequently, $M_{u}$ is never compact if $u \neq 0$.

Corollary 3.4. Let $X\left(\mathbb{B}_{n}\right)$ be any of the following spaces

(a) $A_{\alpha, \beta}^{p}\left(\mathbb{B}_{n}\right), p \geq 1, \beta \geq 0$, and $\alpha>-1$;

(b) $\mathcal{B}_{\alpha}\left(\mathbb{B}_{n}\right), \alpha>0$;

(c) $H_{\beta}^{2}\left(\mathbb{B}_{n}\right), \beta \geq 0$;

(d) $H_{w}^{p}(\mathbb{D}), p>1, w \in\left(A^{p}\right)$.

Then the spectrum of a multiplication operator $M_{u}: X\left(\mathbb{B}_{n}\right) \rightarrow X\left(\mathbb{B}_{n}\right)$ is given by $\sigma\left(M_{u}\right)=$ $\overline{u\left(\mathbb{B}_{n}\right)}$.

\section{The essential spectrum of $M_{u}$}

Examining the essential spectrum of a multiplication operator when the domain is $\mathbb{B}_{n}$, $n>1$, the result concerning $H_{\beta}^{2}$, obtained by Cao, He and $\mathrm{Zhu}$, can be made quite general, see Theorem 4.1. In the case $n=1$, we have obtained a sufficient condition for Fredholmness in Lemma 4.4, where all four conditions (I)-(IV) were assumed. For the spaces mentioned in our main result, namely Theorem 4.13, this condition is also necessary for Fredholmness, see Lemmas 4.9 and 4.12 , but for this to be proved, spacespecific properties were used. An asymptotic approximation for the behaviour of the norm of the peak functions is necessary for the result concerning Bergman-Sobolev spaces. The 
estimate given in Lemma 11 in [10] is insufficient for our purposes, not only because it only considers $p=2$, but also because it is not a sharp lower bound. The necessity of an asymptotic approximation instead of a non-sharp lower bound of the behaviour is clear when an arbitrary $p \in(1, \infty)$ is considered in Lemma 4.12 .

Theorem 4.1. Assume that condition (I) is satisfied and $n>1$. Furthermore, let $u \in$ $M\left(X\left(\mathbb{B}_{n}\right)\right)$ and $P_{j}: \mathbb{B}_{n} \rightarrow \mathbb{C}, P_{j}(z)=z_{j}$ for every $j=1, \ldots, n$. Suppose that $P_{j} \in$ $M\left(X\left(\mathbb{B}_{n}\right)\right)$ for every $j$. Then $\sigma_{e}\left(M_{u}\right)=\bigcap_{0<r<1} \overline{u\left(\mathbb{B}_{n} \backslash r \mathbb{B}_{n}\right)}=\overline{u\left(\mathbb{B}_{n}\right)}=\sigma\left(M_{u}\right)$.

Proof. Let $\lambda \in u\left(\mathbb{B}_{n}\right)$. Since $n>1$, the function $u(z)-\lambda$ has infinitely many distinct zeros, and therefore, there must exist an infinite subset $\left\{\alpha_{k}\right\}_{k=1}^{\infty}, \alpha_{k}=\left(\alpha_{k, 1}, \ldots, \alpha_{k, n}\right)$, of these zeros such that for some $j=1, \ldots, n$ we have $\alpha_{k, j} \neq \alpha_{l, j}$ whenever $k \neq l$. We first show, by induction, that $\left(\delta_{\alpha_{k}}\right)_{k=1}^{\infty}$ are linearly independent in Ker $M_{u-\lambda}^{*}$. Clearly all $\delta_{\alpha_{k}} \in \operatorname{Ker} M_{u-\lambda}^{*}$. Suppose that

$$
\sum_{k=1}^{m} c_{k} \delta_{\alpha_{k}}=0
$$

for some $m \in \mathbb{Z}_{\geq 1}$. If $m=1$, it follows that $c_{1}=0$. Assume that $m \geq 2$. For arbitrary $f \in X\left(\mathbb{B}_{n}\right)$ we have by assumption that $P_{j} f \in X\left(\mathbb{B}_{n}\right)$, so

$$
\sum_{k=1}^{m} c_{k} \alpha_{k, j} \delta_{\alpha_{k}}(f)=0 \text { and } \sum_{k=1}^{m} c_{k} \delta_{\alpha_{k}}(f)=0
$$

Hence

$$
\sum_{k=2}^{m} c_{k}\left(\alpha_{k, j}-\alpha_{1, j}\right) \delta_{\alpha_{k}}(f)=\sum_{k=1}^{m} c_{k}\left(\alpha_{k, j}-\alpha_{1, j}\right) \delta_{\alpha_{k}}(f)=0 \quad \text { for all } f \in X\left(\mathbb{B}_{n}\right),
$$

and therefore, by the induction hypothesis, $c_{k}\left(\alpha_{k, j}-\alpha_{1, j}\right)=0$ for all $k=2, \ldots, m$. This implies that $c_{k}=0$ for $k=2, \ldots, m$, and consequently $c_{1}=0$. Then $\operatorname{Ker} M_{u-\lambda}^{*}$ is infinite dimensional so that $M_{u-\lambda}^{*}$, and equivalently $M_{u-\lambda}$, is not Fredholm. It follows that $u\left(\mathbb{B}_{n}\right) \subset \sigma_{e}\left(M_{u}\right)$ and, moreover, that

$$
\bigcap_{0<r<1} \overline{u\left(\mathbb{B}_{n} \backslash r \mathbb{B}_{n}\right)} \subset \overline{u\left(\mathbb{B}_{n}\right)} \subset \sigma_{e}\left(M_{u}\right) \subset \sigma\left(M_{u}\right)
$$

For the converse conclusion, let $\lambda \notin \bigcap_{0<r<1} \overline{u\left(\mathbb{B}_{n} \backslash r \mathbb{B}_{n}\right)}$. Hence, there are $r \in(0,1)$ and $\delta>0$ such that $|\lambda-u(z)| \geq \delta$ for all $r<|z|<1$. Then $v(z)=(u(z)-\lambda)^{-1}$ is holomorphic and bounded on $\mathbb{B}_{n} \backslash r \overline{\mathbb{B}}_{n}$. As in $[10]$, using Hartogs' extension theorem and the identity theorem, we can extend $v$ to a function $\widetilde{v} \in \mathcal{H}\left(\mathbb{B}_{n}\right)$ such that $\widetilde{v}(z)=(u(z)-\lambda)^{-1}$ for all $z \in \mathbb{B}_{n}$, and therefore $\widetilde{v} \in H^{\infty}\left(\mathbb{B}_{n}\right)$. Now $M_{u-\lambda}$ is invertible by Lemma 3.1 , so $\lambda \notin \sigma\left(M_{u}\right)$. 
Remark 4.2. Following the proof of Theorem 4.1 it is clear that $\left(\delta_{\alpha_{k}}\right)_{k=1}^{\infty}$ are linearly independent when $n=1$.

Now we proceed to the case $n=1$.

The following result is based on ideas due to Axler [3] that was carried on in [8]. It holds for all spaces $X(\mathbb{D})$ such that $M(X(\mathbb{D}))=H^{\infty}(\mathbb{D})$.

Lemma 4.3. Assume that condition $(\mathrm{I})$ is satisfied and let $u \in M(X(\mathbb{D}))=H^{\infty}(\mathbb{D})$. If $M_{u}: X(\mathbb{D}) \rightarrow X(\mathbb{D})$ is Fredholm, then there are $r \in(0,1)$ and $\delta>0$ such that $|u(z)| \geq \delta$ for all $r \leq|z|<1$.

Proof. Assume we can find a sequence $\left(z_{n}\right)_{n=1}^{\infty} \subset \mathbb{D}$ with $\left|z_{n}\right| \rightarrow 1$ and $\left|u\left(z_{n}\right)\right| \rightarrow 0$ when $n \rightarrow \infty$. Then we can assume that $\left(z_{n}\right)_{n}$ is an interpolating sequence in $H^{\infty}(\mathbb{D})$ by going to a subsequence if necessary. Therefore, (see, e.g., [2, Ch. 7.3]) there is a constant $M>0$ such that for each $N \in \mathbb{N}$ there is a function $u_{N} \in H^{\infty}(\mathbb{D})$ with

$$
u_{N}\left(z_{n}\right)= \begin{cases}u\left(z_{n}\right) & \text { if } n \geq N \\ 0 & \text { if } n<N\end{cases}
$$

and $\left\|u_{N}\right\|_{\infty} \leq M \sup _{n \geq N}\left|u\left(z_{n}\right)\right|$. Let

$$
Z_{N}=\left\{f \in X(\mathbb{D}): \delta_{z_{n}}(f)=0 \text { for all } n \geq N\right\}
$$

which is a closed subspace of $X(\mathbb{D})$. From Remark 4.2 we know that the $\delta_{z_{n}} \in X(\mathbb{D})^{*}$ are linearly independent, which implies that $Z_{N}^{\perp}$ is infinite-dimensional. Since $\delta_{z_{n}}(u-$ $\left.u_{N}\right)=0$ for all $n \geq N$, we get $M_{u-u_{N}}(X(\mathbb{D})) \subset Z_{N}$. Now $\left(X(\mathbb{D}) / Z_{N}\right)^{*}=Z_{N}^{\perp}$, so $X(\mathbb{D}) / Z_{N}$ is infinite-dimensional. Hence $X(\mathbb{D}) / M_{u-u_{N}}(X(\mathbb{D}))$ is also infinite-dimensional, and $M_{u-u_{N}}: X(\mathbb{D}) \rightarrow X(\mathbb{D})$ is not Fredholm. As $M(X(\mathbb{D}))=H^{\infty}(\mathbb{D})$ and the set of non-Fredholm operators is closed, it follows from

$$
\left\|M_{u-u_{N}}-M_{u}\right\|=\left\|M_{u_{N}}\right\| \leq C\left\|u_{N}\right\|_{\infty} \leq C M \sup _{n \geq N}\left|u\left(z_{n}\right)\right| \rightarrow 0 \quad \text { as } N \rightarrow \infty
$$

that $M_{u}$ is not Fredholm.

Lemma 4.4. Assume that (I)-(IV) are satisfied and let $u \in M(X(\mathbb{D}))$. If there are $r \in(0,1)$ and $\delta>0$ such that $|u(z)| \geq \delta$ for all $r \leq|z|<1$, then $M_{u}: X(\mathbb{D}) \rightarrow X(\mathbb{D})$ is Fredholm.

Proof. By assumption we have that $u$ can have only finitely many zeros $\alpha_{1}, \ldots, \alpha_{n}$ inside $\mathbb{D}$ with multiplicities $m_{1}, \ldots, m_{n}$ respectively. Then for all $z \in \mathbb{D}$,

$$
u(z)=v(z)\left(z-\alpha_{1}\right)^{m_{1}} \cdots\left(z-\alpha_{n}\right)^{m_{n}}=v(z) p(z),
$$


where $v \in \mathcal{H}(\mathbb{D})$ and $1 / v \in H^{\infty}(\mathbb{D})$.

Let us now define the point evaluation maps for derivatives by $\delta_{z}^{(k)}(f)=f^{(k)}(z)$ for all $z \in \mathbb{D}$ and all $k \in \mathbb{Z}_{\geq 0}$. By assumption $(\mathrm{I})$, it holds that $\delta_{z}^{(k)} \in X(\mathbb{D})^{*}$ for all $k$ and $z$. Clearly,

$$
M_{u}(X(\mathbb{D})) \subset \bigcap_{i=1}^{n} \bigcap_{k=0}^{m_{i}-1} \operatorname{Ker} \delta_{\alpha_{i}}^{(k)}
$$

Let $f \in \bigcap_{i=1}^{n} \bigcap_{k=0}^{m_{i}-1} \operatorname{Ker} \delta_{\alpha_{i}}^{(k)}$, so $f^{(k)}\left(\alpha_{i}\right)=0$ for all $i=1, \ldots, n$ and all $k=0, \ldots, m_{i}-1$. Then $\frac{f}{u} \in \mathcal{H}(\mathbb{D})$. Now assumption (IV) implies that $v \in M(X(\mathbb{D}))$. Indeed, if $g \in X(\mathbb{D})$, then $u g \in X(\mathbb{D})$ and by assumption $(\mathrm{IV})$ it follows that $v g=\frac{u g}{p} \in X(\mathbb{D})$. Therefore, $1 / v \in M(X(\mathbb{D}))$ by Lemma 3.1 , so that $\frac{f}{u}=\frac{f / p}{v} \in X(\mathbb{D})$ by assumption (IV). As a result, $f=u \frac{f}{u} \in M_{u}(X(\mathbb{D}))$, and thus

$$
M_{u}(X(\mathbb{D}))=\bigcap_{i=1}^{n} \bigcap_{k=0}^{m_{i}-1} \operatorname{Ker} \delta_{\alpha_{i}}^{(k)}
$$

Consequently, $M_{u}$ has closed range, and since $M_{u}: X(\mathbb{D}) \rightarrow X(\mathbb{D})$ is always injective, the dimension of the kernel of $M_{u}$ is finite. Since ${ }^{\perp}\left(\operatorname{span}\left\{\delta_{\alpha_{i}}^{(k)}\right\}\right)=\operatorname{Ker} \delta_{\alpha_{i}}^{(k)}$, it follows that the $w^{*}$-closed one-dimensional space $\operatorname{span}\left\{\delta_{\alpha_{i}}^{(k)}\right\}=\left(\operatorname{Ker} \delta_{\alpha_{i}}^{(k)}\right)^{\perp}$, see 16, Theorem 11 on p. 341]. Therefore, by [16, Theorem 13 on p. 342], we have

$$
M_{u}(X(\mathbb{D}))^{\perp}=\sum_{i=1}^{n} \sum_{k=0}^{m_{i}-1}\left(\operatorname{Ker} \delta_{\alpha_{i}}^{(k)}\right)^{\perp}=\sum_{i=1}^{n} \sum_{k=0}^{m_{i}-1} \operatorname{span}\left\{\delta_{\alpha_{i}}^{(k)}\right\}
$$

and hence, the dimension of the co-kernel of $M_{u}$ is finite, and $M_{u}$ is Fredholm.

Theorem 4.5. Assume that (I), (III) and (IV) are satisfied and $M(X(\mathbb{D}))=H^{\infty}(\mathbb{D})$. Let $M_{u}: X(\mathbb{D}) \rightarrow X(\mathbb{D})$ be a multiplication operator generated by $u \in M(X(\mathbb{D}))$. The essential spectrum of $M_{u}$ is given by $\sigma_{e}\left(M_{u}\right)=\bigcap_{0<r<1} \overline{u(\mathbb{D} \backslash r \mathbb{D})}$.

Proof. We have that $\lambda \in \overline{u(\mathbb{D} \backslash r \mathbb{D})}$ for all $r \in(0,1)$ if and only if for all $r \in(0,1)$ there is a sequence $\left(z_{n}\right)_{n=1}^{\infty} \subset \mathbb{D}$ such that $\left|z_{n}\right| \geq r$ for all $n \in \mathbb{N}$ and $\left|u\left(z_{n}\right)-\lambda\right| \rightarrow 0$ when $n \rightarrow \infty$. Since $M_{u}-\lambda I=M_{u-\lambda}$, we can now apply Lemmas 4.3 and 4.4 to conclude that the last statement equivalently means that $M_{u}-\lambda I$ is not Fredholm, that is $\lambda \in \sigma_{e}\left(M_{u}\right)$. The use of Lemma 4.4 is justified by the remark after condition (II).

In Example 2.2 2.4 they were stated that the multiplier spaces for $A_{\alpha, \beta}^{p}(\mathbb{D})$ with $p \geq 1$, $\alpha>-1, \beta<\frac{1+\alpha}{p} ; H_{w}^{p}(\mathbb{D})$ with $p>1, w \in\left(A^{p}\right)$ and $\mathcal{B}_{\alpha}(\mathbb{D})$ with $\alpha>1$ are $H^{\infty}(\mathbb{D})$. Thus, we obtain the following results.

Corollary 4.6. In each of the following three cases: 
(a) $p \geq 1, \alpha>-1$ and $\beta<\frac{1+\alpha}{p}$ with $u \in M\left(A_{\alpha, \beta}^{p}(\mathbb{D})\right)$;

(b) $\alpha>1$ with $u \in M\left(\mathcal{B}_{\alpha}(\mathbb{D})\right)$;

(c) $p>1, w \in\left(A^{p}\right)$ with $u \in M\left(H_{w}^{p}(\mathbb{D})\right)$,

the essential spectrum of $M_{u}$ is given by

$$
\sigma_{e}\left(M_{u}\right)=\bigcap_{0<r<1} \overline{u(\mathbb{D} \backslash r \mathbb{D})} .
$$

It was shown in Theorem 4.1 that in higher dimensions, $n>1$, the essential spectra of multiplication operators coincide with their spectra for many spaces. This is seldom true for $n=1$. In Corollaries 3.4 and 4.6 and Theorem 4.13 , we list some spaces, on which multiplier operators have the spectrum given by $\overline{u\left(\mathbb{B}_{n}\right)}$ and the essential spectrum given by $\bigcap_{0<r<1} \overline{u\left(\mathbb{B}_{n} \backslash r \mathbb{B}_{n}\right)}$. Although the sets may differ, their spectral and essential spectral radii coincide according to the following remark.

Remark 4.7. (a) Let $n \in \mathbb{Z}_{\geq 1}$. Since the decreasing sequence $\left(\overline{u\left(\mathbb{B}_{n} \backslash\left(1-\frac{1}{k}\right) \mathbb{B}_{n}\right)}\right)_{k=2}^{\infty}$ consists of compact and connected sets, the intersection $\bigcap_{0<r<1} \overline{u\left(\mathbb{B}_{n} \backslash r \mathbb{B}_{n}\right)}$ is compact and connected.

(b) For $n \in \mathbb{Z}_{\geq 1}$ and $u \in H^{\infty}\left(\mathbb{B}_{n}\right)$ we have

$$
\sup \left\{|\lambda|: \lambda \in \bigcap_{0<r<1} \overline{u\left(\mathbb{B}_{n} \backslash r \mathbb{B}_{n}\right)}\right\}=\|u\|_{\infty}=\sup _{\lambda \in \overline{u\left(\mathbb{B}_{n}\right)}}|\lambda| .
$$

Moreover, both suprema are attained. Clearly

$$
\|u\|_{\infty}=\sup _{z \in \mathbb{B}_{n}}|u(z)|=\sup _{\lambda \in u\left(\mathbb{B}_{n}\right)}|\lambda|=\sup _{\lambda \in \overline{u\left(\mathbb{B}_{n}\right)}}|\lambda| \geq \sup \left\{|\lambda|: \lambda \in \bigcap_{0<r<1} \overline{u\left(\mathbb{B}_{n} \backslash r \mathbb{B}_{n}\right)}\right\} .
$$

Furthermore, since $u \in H^{\infty}$ there is a sequence $\left(z_{j}\right)_{j=1}^{\infty}$ such that $z_{j} \in \mathbb{B}_{n} \backslash r_{j} \mathbb{B}_{n}$ and $\lim _{j \rightarrow \infty}\left|u\left(z_{j}\right)\right|=\|u\|_{\infty}$, where $r_{j}=1-j^{-1}$. The sequence $\left(u\left(z_{j}\right)\right)_{j=1}^{\infty}$ is bounded, and therefore, by Bolzano-Weierstrass theorem, there is a convergent subsequence $\left(\lambda_{k}\right)_{k=1}^{\infty}$, where $\lambda_{k}=u\left(z_{j_{k}}\right) \in \overline{u\left(\mathbb{B}_{n} \backslash r_{j_{k}} \mathbb{B}_{n}\right)}$. Since the sets $U_{k}=\overline{u\left(\mathbb{B}_{n} \backslash r_{j_{k}} \mathbb{B}_{n}\right)}$ are compact and $U_{k+1} \subset U_{k}, k=1,2, \ldots$, it holds that $\lim _{k \rightarrow \infty} \lambda_{k}=\lambda \in U_{j}$ for every $j$, and hence, we have $\lambda \in \bigcap_{0<r<1} \overline{u\left(\mathbb{B}_{n} \backslash r \mathbb{B}_{n}\right)}$ and $|\lambda|=\|u\|_{\infty}$.

For $\xi \in \partial \mathbb{D}$ and $k \in \mathbb{Z}_{\geq 1}$, let $f_{\xi, k}: \mathbb{D} \rightarrow \mathbb{D}$ be a peak function defined by

$$
f_{\xi, k}(z)=\left(\frac{1+\bar{\xi} z}{2}\right)^{k}
$$

For $\alpha>0$ it is well-known that $\mathcal{B}_{0, \alpha}(\mathbb{D})^{*} \simeq A_{0}^{1}(\mathbb{D})$ and $A_{0}^{1}(\mathbb{D})^{*} \simeq \mathcal{B}_{\alpha}(\mathbb{D})$ via an integral pairing, see 21. 
Lemma 4.8. Let $0<\alpha \leq 1, \xi \in \mathbb{D}$, and $g_{\xi, k}(z)=\left(\frac{1+\bar{\xi} z}{2}\right)^{k}\left\|\left(\frac{1+\bar{\xi} z}{2}\right)^{k}\right\|_{\mathcal{B}_{\alpha}}^{-1}$ be the normalized peak function. Then we have $g_{\xi, k}^{(m)} \rightarrow 0, m \in \mathbb{Z}_{\geq 0}$ uniformly on every set $A_{\delta}=\{z \in \mathbb{D}$ : $|z-\xi| \geq \delta\}, \delta>0$, and $g_{\xi, k} \rightarrow 0$ weakly in $\mathcal{B}_{\alpha}(\mathbb{D})$ as $k \rightarrow \infty$.

Proof. For the Bloch-type spaces $\mathcal{B}_{\alpha}(\mathbb{D})$, it can be shown that

$$
\left\|\left(\frac{1+\bar{\xi} z}{2}\right)^{k}\right\|_{\mathcal{B}_{\alpha}} \asymp k^{1-\alpha} .
$$

The property $g_{\xi, k}^{(m)} \rightarrow 0, m \in \mathbb{Z}_{\geq 0}$, uniformly on the sets $A_{\delta}$ as $k \rightarrow \infty$ is a consequence of the definition of $g_{\xi, k}$. Moreover, the sequence $\left(g_{\xi, k}\right)_{k=1}^{\infty}$ is a weak* null sequence by using Lemma 3.1 in $[12]$. Since $\left(g_{\xi, k}\right)_{k} \subset \mathcal{P}(\mathbb{D}) \subset \mathcal{B}_{0, \alpha}(\mathbb{D})$, we conclude that $g_{\xi, k} \rightarrow 0$ weakly when $k \rightarrow \infty$.

Lemma 4.9. Let us assume that either $u \in M(\mathcal{B}(\mathbb{D})) \cap A(\mathbb{D})$ or $u \in M\left(\mathcal{B}_{\alpha}(\mathbb{D})\right)=\mathcal{B}_{\alpha}(\mathbb{D})$ with $0<\alpha<1$. If $M_{u}: \mathcal{B}_{\alpha}(\mathbb{D}) \rightarrow \mathcal{B}_{\alpha}(\mathbb{D})$ is Fredholm, then there are $r \in(0,1)$ and $\delta>0$ such that $|u(z)| \geq \delta$ for all $r \leq|z|<1$.

Proof. Suppose there is a sequence $\left(z_{k}\right)_{k=1}^{\infty} \subset \mathbb{D}$ such that $\left|z_{k}\right| \rightarrow 1$ and $\left|u\left(z_{k}\right)\right| \rightarrow 0$ when $k \rightarrow \infty$. Then, by going to a subsequence if necessary, we can assume that $z_{k} \rightarrow \xi \in \partial \mathbb{D}$ when $k \rightarrow \infty$. Since $u$ is continuous up to the boundary in both cases, $u(\xi)=0$. Now by Lemma 4.8 it holds that $g_{\xi, k} \rightarrow 0, g_{\xi, k}^{\prime} \rightarrow 0$ uniformly on every set $A_{\delta}=\{z \in \mathbb{D}:|z-\xi| \geq$ $\delta\}, \delta>0$, and $g_{\xi, k} \rightarrow 0$ weakly as $k \rightarrow \infty$. We consider the two cases: (i) when $\alpha=1$ and (ii) when $0<\alpha<1$.

(i) It holds that $\sup _{k \in \mathbb{Z}_{>1}}\left\|g_{\xi, k}\right\|_{\infty}<\infty$. Since $u \in M(\mathcal{B}(\mathbb{D}))$, we know that $u \in$ $\mathcal{B}_{0}(\mathbb{D}) \cap H^{\infty}(\mathbb{D})$. Let $B_{\delta}=\{z \in \mathbb{D}:|z-\xi|<\delta\}$, so $\mathbb{D}=A_{\delta} \cup B_{\delta}$. Let $\varepsilon>0$ be given, and choose $\delta>0$ such that $|u(z)|<\varepsilon$ and $\left|u^{\prime}(z)\right|\left(1-|z|^{2}\right)<\varepsilon$ for $z \in B_{\delta}$. The following estimates hold,

$$
\left\|M_{u}\left(g_{\xi, k}\right)\right\|_{\mathcal{B}} \leq I_{k, A_{\delta}}+I I_{k, B_{\delta}}+\left|g_{\xi, k}(0) u(0)\right|
$$

where

$$
\begin{aligned}
I_{k, A_{\delta}} & =\sup _{z \in A_{\delta}}|u(z)|\left|g_{\xi, k}^{\prime}(z)\right|\left(1-|z|^{2}\right)+\sup _{z \in A_{\delta}}\left|u^{\prime}(z)\right|\left|g_{\xi, k}(z)\right|\left(1-|z|^{2}\right), \\
I I_{k, B_{\delta}} & =\sup _{z \in B_{\delta}}|u(z)|\left|g_{\xi, k}^{\prime}(z)\right|\left(1-|z|^{2}\right)+\sup _{z \in B_{\delta}}\left|u^{\prime}(z)\right|\left|g_{\xi, k}(z)\right|\left(1-|z|^{2}\right) .
\end{aligned}
$$

Consequently, we get that $\lim _{k \rightarrow \infty} I_{k, A_{\delta}}=0$ and $\lim _{k \rightarrow \infty} I I_{k, B_{\delta}} \leq 2 \varepsilon$. We also have $\left|g_{\xi, k}(0)\right| \asymp 2^{-k}$. Thus $\left\|M_{u}\left(g_{\xi, k}\right)\right\|_{\mathcal{B}} \rightarrow 0$ when $k \rightarrow \infty$, which means by Lemma 4.3 .15 in [13] that $0 \in \sigma_{e}\left(M_{u}\right)$. Therefore $M_{u}$ is not Fredholm.

(ii) The result follows similarily from showing that $\left\|u g_{\xi, k}\right\|_{\mathcal{B}_{\alpha}} \rightarrow 0$ as $k \rightarrow \infty$. Take $\varepsilon>0$ and choose $\delta>0$ such that $|u(z)|<\varepsilon$ on $B_{\delta}$. It is clear that $I_{k, A_{\delta}} \rightarrow 0$ as $k \rightarrow \infty$. 
From the definition of $g_{\xi, k}$ we have $\left\|g_{\xi, k}\right\|_{\infty} \asymp k^{\alpha-1}$, so $\left\|g_{\xi, k}\right\|_{\infty} \rightarrow 0$ as $k \rightarrow \infty$, hence, $I I_{k, B_{\delta}}<2 \varepsilon$ for $k$ large enough.

Let us now consider the space $X(\mathbb{D})=A_{\alpha, \beta}^{p}(\mathbb{D})$ with $1<p<\infty$. The following lemma will be used to obtain an estimate for the Bergman-Sobolev norm of the peak function.

Lemma 4.10. Let $L, M \geq 0$. Then

$$
\frac{\Gamma(K+L)}{\Gamma(K)} \sim K^{L} \quad \text { and } \quad \frac{\Gamma(2 K+L)}{\Gamma(K+L) \Gamma(K+M)} \sim \frac{2^{2 K+L-1}}{\sqrt{\pi}} K^{1 / 2-M}
$$

as $K \rightarrow \infty$.

Proof. According to Stirling's approximation, $\Gamma(x) \sim \sqrt{\frac{2 \pi}{x}}\left(\frac{x}{e}\right)^{x}$ as $x \rightarrow \infty$, we have

$$
\begin{aligned}
\frac{\Gamma(K+L)}{K^{L} \Gamma(K)} & \sim \sqrt{\frac{K}{K+L}} e^{K-(K+L)} \frac{(K+L)^{K+L}}{K^{L} K^{K}} \\
& =\left(1+\frac{L}{K}\right)^{-1 / 2} e^{-L}\left(1+\frac{L}{K}\right)^{K}\left(1+\frac{L}{K}\right)^{L} \rightarrow 1
\end{aligned}
$$

as $K \rightarrow \infty$. Moreover,

$$
\begin{aligned}
& \frac{\sqrt{\pi} K^{M-1 / 2} \Gamma(2 K+L)}{2^{L+2 K-1} \Gamma(K+L) \Gamma(K+M)} \\
\sim & \frac{\sqrt{\pi} K^{M-1 / 2}}{2^{L+2 K-1}} \sqrt{\frac{(K+L)(K+M)}{2 \pi(2 K+L)}} \frac{e^{M}(2 K+L)^{2 K+L}}{(K+L)^{K+L}(K+M)^{K+M}} \\
= & e^{M} \sqrt{\frac{\left(1+\frac{L}{K}\right)\left(1+\frac{M}{K}\right)}{\left(1+\frac{L}{2 K}\right)} \frac{\left(1+\frac{L}{2 K}\right)^{2 K+L}}{\left(1+\frac{L}{K}\right)^{K+L}\left(1+\frac{M}{K}\right)^{K+M}} \rightarrow 1}
\end{aligned}
$$

as $K \rightarrow \infty$.

In the following important lemma a fairly good approximation of the behaviour of the Bergman-Sobolev norm of the peak functions is obtained. The proof also gives an exact asymptotic formula for $\left\|D^{j} f_{\xi, k}\right\|_{A_{\alpha}^{p}}$ as $k \rightarrow \infty$ in the case of $p \in \mathbb{Z}_{\geq 1}$, namely,

$$
\left\|D^{j} f_{\xi, k}\right\|_{A_{\alpha}^{p}}^{p} \sim \frac{\Gamma(\alpha+2) 2^{2 \alpha+5 / 2-j p}}{\sqrt{\pi} p^{\alpha+3 / 2}}(k+1)^{j p-(\alpha+3 / 2)} .
$$

Furthermore, some properties for the normalized peak function are given in order to prove Lemma 4.12, from which a part of the main result follows. Observe that, as already mentioned in the beginning of Section 4 , the following lemma is a necessary refinement of Lemma 11 in 10 and, as a sharp estimate, it is also of independent interest. 
Lemma 4.11. Let $p \geq 1, \alpha>-1$ or $p=2, \alpha=-1$. If $\beta \geq 0$, then

$$
\left\|f_{\xi, k}\right\|_{A_{\alpha, \beta}^{p}}^{p} \asymp(k+1)^{-\alpha+\beta p-3 / 2}
$$

for $k \in \mathbb{Z}$ large enough. Consequently, if $\beta>\frac{2+\alpha}{p}$ and $\xi \in \partial \mathbb{D}$, then the functions $g_{\xi, k}=$ $f_{\xi, k} /\left\|f_{\xi, k}\right\|_{A_{\alpha, \beta}^{p}} \in \mathcal{P}(\mathbb{D})$ have the properties that $\left\|g_{\xi, k}\right\|_{A_{\alpha, \beta}^{p}}=1 ; g_{\xi, k} \rightarrow 0 ; R^{m} g_{\xi, k} \rightarrow 0$, $m \in \mathbb{Z}_{\geq 1}$, uniformly on every set $A_{\delta}=\{z \in \mathbb{D}:|z-\xi| \geq \delta\}, \delta>0$, and for $p>1$ it also holds that $g_{\xi, k} \rightarrow 0$ weakly in $A_{\alpha, \beta}^{p}$ as $k \rightarrow \infty$.

Proof. Let $N$ be a positive integer satisfying $N>\beta-\frac{1 / 2+\alpha}{p}$. By 2.2 and 2.3 we have that

$$
\begin{aligned}
\left\|f_{\xi, k}\right\|_{A_{\alpha, \beta}^{p}} & \asymp\left\|f_{\xi, k}\right\|_{A_{(N-\beta) p+\alpha, N}^{p}} \asymp \sum_{l=0}^{N-1}\left|D^{l} f_{\xi, k}(0)\right|+\left\|D^{N} f_{\xi, k}\right\|_{A_{(N-\beta) p+\alpha}^{p}} \\
& \asymp\left\|D^{N} f_{\xi, k}\right\|_{A_{(N-\beta) p+\alpha}^{p}} .
\end{aligned}
$$

The last equivalence follows from

$$
0 \leq\left|D^{l} f_{\xi, k}(0)\right| \leq\left|D^{N} f_{\xi, k}(0)\right| \leq\left\|D^{N} f_{\xi, k}\right\|_{A_{(N-\beta) p+\alpha}^{p}}
$$

for $l \leq N$. To finish the proof, it will be shown that for $\gamma>-1$ and $j \in \mathbb{Z}_{\geq 0}$ we have

$$
\left\|D^{j} f_{\xi, k}\right\|_{A_{\gamma}^{p}}^{p} \asymp(k+1)^{j p-(\gamma+3 / 2)},
$$

from which the lemma follows by letting $\gamma=(N-\beta) p+\alpha$ and $j=N$.

Let $q$ be the smallest integer greater than or equal to $p$ and $k \geq j$. We have

$$
\begin{aligned}
\frac{\left\|D^{j} f_{\xi, k}\right\|_{A_{\gamma}^{p}}^{p}}{\gamma+1} & =\int_{\mathbb{D}}\left|D^{j} f_{\xi, k}(z)\right|^{p}\left(1-|z|^{2}\right)^{\gamma} d A(z) \\
& =\left(\frac{k !}{(k-j) !}\right)^{p} \int_{\mathbb{D}}\left(\frac{|1+\bar{\xi} z|^{(k-j)}}{2^{k}}\right)^{p}\left(1-|z|^{2}\right)^{\gamma} d A(z) \\
& \stackrel{(*)}{\geq}\left(\frac{k !}{(k-j) !}\right)^{p} \int_{\mathbb{D}}\left(\frac{|1+\bar{\xi} z|^{(k-j)}}{2^{k}}\right)^{q}\left(1-|z|^{2}\right)^{\gamma} d A(z) \\
& \stackrel{(* *)}{\geq}\left(\frac{k !}{(k-j) !}\right)^{p} \int_{\mathbb{D}} \frac{1}{2^{j q}} \frac{|1+\bar{\xi} z|^{2 K}}{2^{2 K}}\left(1-|z|^{2}\right)^{\gamma} d A(z)=U_{k, \xi, j, \gamma} .
\end{aligned}
$$

The $(*)$ indicates that choosing $q$ to be the greatest integer smaller than $p$ we similarily obtain the opposite strict inequality. The function $K: \mathbb{Z}_{\geq j} \rightarrow \mathbb{Z}_{\geq 0}$ is defined as $K=$ $K_{j}(k)=\frac{(k-j) q}{2}$ if $k-j$ is even. In this case $\stackrel{(* *)}{\geq}$ is an equality. If $k-j$ is odd, then $K$ is defined by $K=\frac{(k+1-j) q}{2}$ or $K=\frac{(k-1-j) q}{2}$ depending on which inequality we want to obtain. In the latter case $\stackrel{(* *)}{\geq}$ is replaced by $\leq$. 
We continue the proof by evaluating the integral with respect to the angle. It is enough to examine the expression for $\xi=1$. Now consider the functions $g_{r} \in L^{2}([0,2 \pi))$, $g_{r}(t)=\left(1+r e^{i t}\right)^{K}=\sum_{n=0}^{K}\left(\begin{array}{c}K \\ n\end{array}\right) r^{n} e^{i t n}$ for $r \geq 0$. From Parseval's equality we obtain

$$
\int_{0}^{2 \pi}\left|1+r e^{i t}\right|^{2 K} d t=2 \pi \sum_{n=0}^{K}\left(\begin{array}{c}
K \\
n
\end{array}\right)^{2} r^{2 n},
$$

for every $0 \leq r<1$ and thus,

$$
\begin{aligned}
U_{k, \xi, j, \gamma} & =\left(\frac{k !}{(k-j) !}\right)^{p} \frac{2}{2^{(j q+2 K)}} \int_{0}^{1} \sum_{n=0}^{K}\left(\begin{array}{c}
K \\
n
\end{array}\right)^{2} r^{2 n}\left(1-r^{2}\right)^{\gamma} r d r \\
& =\left(\frac{k !}{(k-j) !}\right)^{p} \frac{1}{2^{(j q+2 K)}} \sum_{n=0}^{K}\left(\begin{array}{c}
K \\
n
\end{array}\right)^{2} \int_{0}^{1} r^{2 n}\left(1-r^{2}\right)^{\gamma} 2 r d r \\
& =\left(\frac{k !}{(k-j) !}\right)^{p} \frac{1}{2^{(j q+2 K)}} \sum_{n=0}^{K}\left(\begin{array}{c}
K \\
n
\end{array}\right)^{2} \int_{0}^{1} r^{n}(1-r)^{\gamma} d r .
\end{aligned}
$$

Moreover,

$$
\begin{aligned}
U_{k, \xi, j, \gamma} & =\left(\frac{k !}{(k-j) !}\right)^{p} \frac{1}{2^{(j q+2 K)}} \sum_{n=0}^{K}\left(\begin{array}{c}
K \\
n
\end{array}\right)^{2} \beta(n+1, \gamma+1) \\
& =\left(\frac{k !}{(k-j) !}\right)^{p} \frac{1}{2^{(j q+2 K)}} \sum_{n=0}^{K}\left(\begin{array}{c}
K \\
n
\end{array}\right)^{2} \frac{\Gamma(\gamma+1) \Gamma(n+1)}{\Gamma(n+\gamma+2)} \\
& =\left(\frac{\Gamma(k-j+1+j)}{\Gamma(k-j+1)}\right)^{p} \frac{1}{2^{(j q+2 K)}} \frac{\Gamma(\gamma+1)}{\Gamma(K+\gamma+2)} \frac{\Gamma(2 K+\gamma+2)}{\Gamma(K+\gamma+2)} \\
& \sim k^{j p} \frac{\Gamma(\gamma+1)}{2^{(j q+2 K)} K^{-\gamma-3 / 2}} \frac{2^{\gamma+1+2 K}}{\sqrt{\pi}} \\
& \sim \Gamma(\gamma+1) k^{j p}(k q)^{-\gamma-3 / 2} \frac{2^{2 \gamma+5 / 2-j q}}{\sqrt{\pi}} \\
& =\frac{\Gamma(\gamma+1) 2^{2 \gamma+5 / 2-j q}}{\sqrt{\pi} q^{\gamma+3 / 2}} k^{j p-(\gamma+3 / 2)}
\end{aligned}
$$

as $k \rightarrow \infty$, where the first asymptotic approximation is given by Lemma 4.10 and $(k-c)^{a} \sim$ $k^{a}$ as $k \rightarrow \infty$ for every $c \in \mathbb{R}$. The third equality follows from the Chu-Vandermonde identity, see [15, p. 32] with the parameters $n=K, b=-K$ and $c=\gamma+2$.

To prove that $\left(g_{\xi, k}\right)_{k=1}^{\infty}$ is a weak null sequence, let $B_{A_{\alpha, \beta}^{p}(\mathbb{D})}$ denote the closed unit ball of the Bergman-Sobolev space $A_{\alpha, \beta}^{p}(\mathbb{D}), p>1$. Let $\tau_{p}$ denote the topology of pointwise convergence. Notice that $\left(B_{A_{\alpha, \beta}^{p}(\mathbb{D})}, \tau_{p}\right)$ is a Hausdorff space and that $B_{A_{\alpha, \beta}^{p}(\mathbb{D})}$ is weakly compact, since the space is reflexive. Since $\delta_{z} \in A_{\alpha, \beta}^{p}(\mathbb{D})^{*}$ by condition $(\mathrm{I})$, the identity map

$$
\operatorname{id}:\left(B_{A_{\alpha, \beta}^{p}(\mathbb{D})}, w\right) \rightarrow\left(B_{A_{\alpha, \beta}^{p}(\mathbb{D})}, \tau_{p}\right)
$$


is continuous, and hence, it represents a homeomorphism between the spaces $\left(B_{A_{\alpha, \beta}^{p}(\mathbb{D})}, w\right)$ and $\left(B_{A_{\alpha, \beta}^{p}(\mathbb{D})}, \tau_{p}\right)$. Since $\mathrm{id}^{-1}:\left(B_{A_{\alpha, \beta}^{p}(\mathbb{D})}, \tau_{p}\right) \rightarrow\left(B_{A_{\alpha, \beta}^{p}(\mathbb{D})}, w\right)$ is continuous, we conclude that $g_{\xi, k} \rightarrow 0$ weakly, when $k \rightarrow \infty$.

Lemma 4.12. Let $\alpha>-1, p>1$ or $\alpha=-1, p=2$ and assume $\beta>\frac{2+\alpha}{p}$. If $M_{u}: A_{\alpha, \beta}^{p} \rightarrow$ $A_{\alpha, \beta}^{p}$ is Fredholm, then there exist $\delta>0$ and $r \in(0,1)$ such that $|u(z)| \geq \delta$ for all $r \leq|z|<1$.

Proof. The proof will be carried out by contraposition. Since $u$ belongs to the disk algebra it is continuous up to the boundary of $\mathbb{D}$. Assume there is a point $\xi \in \partial \mathbb{D}$ such that $u(\xi)=0$. This assumption is equivalent to $u$ not being bounded from below arbitrarily close to the boundary, since $u$ is continuous. It will be shown that

$$
\left\|u g_{\xi, k}\right\|_{A_{p(N-\beta)+\alpha, N}^{p}} \rightarrow 0 \quad \text { as } k \rightarrow \infty
$$

which by 2.2 implies that

$$
\left\|u g_{\xi, k}\right\|_{A_{\alpha, \beta}^{p}} \rightarrow 0 \quad \text { as } k \rightarrow \infty
$$

where $N$ is the positive integer satisfying

$$
0<N-\beta+\frac{1 / 2+\alpha}{p} \leq 1,
$$

and $g_{\xi, k}$ is the function defined in Lemma 4.11. The lemma follows from Lemma 4.11. (4.1) and Lemma 4.3.15 in 13 .

To prove the null sequence statement, we will make use of 2.4. First, notice that by Lemma 4.11 we obtain

$$
\left|u(0) g_{\xi, k}(0)\right| \lesssim \frac{\left|u(0) f_{\xi, k}(0)\right|}{(k+1)^{-\frac{\alpha}{p}+\beta-\frac{3}{2 p}}} \rightarrow 0
$$

as $k \rightarrow \infty$. Using the general Leibniz formula we have

$$
R^{N}\left(u g_{\xi, k}\right)=\sum_{j=0}^{N}\left(\begin{array}{c}
N \\
j
\end{array}\right) R^{j} u R^{N-j} g_{\xi, k},
$$

from which it follows that

$$
\left\|R^{N}\left(u g_{\xi, k}\right)\right\|_{A_{p(N-\beta)+\alpha}^{p}} \leq \sum_{j=0}^{N}\left(\begin{array}{c}
N \\
j
\end{array}\right)\left\|R^{j} u R^{N-j} g_{\xi, k}\right\|_{A_{p(N-\beta)+\alpha}^{p}} .
$$

Therefore, it suffices to show that

$$
I_{k, j}=\int_{\mathbb{D}}\left|R^{j} u R^{N-j} g_{\xi, k}\right|^{p} d A_{p(N-\beta)+\alpha}
$$


approaches zero for $j=0,1, \ldots, N$ as $k$ tends to infinity. To prove the assertion for the case $j=0$, we take $\varepsilon>0$ and choose $\delta>0$ such that $|u(z)|^{p}<\varepsilon$ for all

$$
z \in B_{\delta}=\{z \in \mathbb{D}:|z-\xi|<\delta\}
$$

We can now choose a $K>0$ such that

$$
\int_{A_{\delta}}\left|R^{N} g_{\xi, k}(z)\right|^{p} d A_{p(N-\beta)+\alpha}(z)<\varepsilon,
$$

which implies

$$
\int_{A_{\delta}}\left|u(z) R^{N} g_{\xi, k}(z)\right|^{p} d A_{p(N-\beta)+\alpha}(z)<\|u\|_{\infty}^{p} \varepsilon
$$

for $k>K$, where Lemma 4.11 has been used and $A_{\delta}=\{z \in \mathbb{D}:|z-\xi| \geq \delta\}$. Thus, for $k>K$

$$
I_{k, 0}<\left(\|u\|_{\infty}^{p}+\left\|R^{N} g_{\xi, k}\right\|_{A_{p(N-\beta)+\alpha}^{p}}^{p}\right) \varepsilon \leq\left(\|u\|_{\infty}^{p}+M\left\|g_{\xi, k}\right\|_{A_{\alpha, \beta}^{p}}^{p}\right) \varepsilon
$$

where 2.2 gives the second inequality for some $M>0$. Since $u \in A_{\alpha, \beta}^{p}(\mathbb{D}) \subset H^{\infty}(\mathbb{D})$ and $\left\|g_{\xi, k}\right\|_{A_{\alpha, \beta}^{p}}=1$ for every $k$, the result follows. To assure the result in the case $j \geq 1$, we will use the following approximation:

$$
I_{k, j} \leq \frac{(k+1)^{p(N-j)}}{\left\|f_{\xi, k}\right\|_{A_{\alpha, \beta}^{p}}^{p}} \int_{\mathbb{D}}\left|R^{j} u(z)\right|^{p}\left|\frac{1+\bar{\xi} z}{2}\right|^{(k-(N-j)) p} d A_{p(N-\beta)+\alpha}(z) .
$$

From Lemma 4.11 it follows that

$$
\begin{aligned}
I_{k, j} & \lesssim \frac{(k+1)^{p(N-j)}}{(k+1)^{-\alpha+\beta p-3 / 2}} \int_{\mathbb{D}}\left|R^{j} u(z)\right|^{p}\left|\frac{1+\bar{\xi} z}{2}\right|^{(k-(N-j)) p}\left(1-|z|^{2}\right)^{p(N-\beta)+\alpha} d A(z) \\
& =(k+1)^{p\left(N-\beta+\frac{\alpha+3 / 2}{p}-j\right)} \int_{\mathbb{D}}\left|R^{j} u(z)\right|^{p}\left|\frac{1+\bar{\xi} z}{2}\right|^{(k-(N-j)) p}\left(1-|z|^{2}\right)^{p(N-\beta)+\alpha} d A(z) .
\end{aligned}
$$

For integers $j \in[2, N]$ the result $I_{k, j} \rightarrow 0$ as $k \rightarrow \infty$ is obtained from the following three facts:

$$
\begin{gathered}
u \in A_{\alpha, \beta}^{p} \simeq A_{p(N-\beta)+\alpha, N}^{p} \subset A_{p(N-\beta)+\alpha, j}^{p}, \\
\left\|f_{\xi, k}\right\|_{\infty} \leq 1 \quad \forall k \in \mathbb{Z}_{\geq 1}, \\
p\left(N-\beta+\frac{3 / 2+\alpha}{p}-j\right) \leq 1+p-p j<0 .
\end{gathered}
$$

For $j=1$ we make an additional partition. We will, once at a time, assume that $N-\beta+\frac{3 / 2+\alpha}{p}-1$ is strictly less than zero, equal to zero or strictly larger than zero. In the first case we can apply the procedure used for $j \geq 2$. In the second case we may utilize the Lebesgue dominated convergence theorem to functions

$$
\left|\frac{1+\bar{\xi} z}{2}\right|^{(k-(N-j)) p} \leq 1
$$


for all $z \in \mathbb{D}$ and $k \in \mathbb{Z}_{\geq N}$ to obtain the result.

The only thing that remains to show is that $I_{k, 1} \rightarrow 0$ as $k \rightarrow \infty$ when $N-\beta+\frac{3 / 2+\alpha}{p}-1>$ 0 . This condition implies that

$$
N>\beta-\frac{3 / 2+\alpha}{p}+1>1,
$$

so that $N \geq 2$.

To prove that $\left(I_{k, 1}\right)_{k=1}^{\infty}$ is a null sequence we will use Lemma 5.4 in 4 and Lemma 4.11 . Lemma 5.4 in 4 gives us three different approximations for the behaviour of $|D u(z)|$, depending on values of some parameters. Hence, it suffices to prove the null convergence for all of these approximations, one at a time. Notice that $q=\alpha+1$ when comparing notations with [4]. First, assume $\beta<\frac{2+\alpha}{p}+1$. Then we have

$$
\begin{aligned}
I_{k, 1} & \lesssim\|u\|_{A_{\alpha, \beta}^{p}}^{p} \int_{\mathbb{D}}\left|R^{N-1} g_{\xi, k}\right|^{p}\left(1-|z|^{2}\right)^{-p\left(\frac{2+\alpha}{p}+1-\beta\right)} d A_{p(N-\beta)+\alpha}(z) \\
& \lesssim \frac{\|u\|_{A_{\alpha, \beta}^{p}}^{p}}{\left\|f_{\xi, k}\right\|_{A_{\alpha, \beta}^{p}}^{p}} \int_{\mathbb{D}}\left|R^{N-1} f_{\xi, k}\right|^{p} d A_{p(N-1)-2}(z) \\
& =\frac{\|u\|_{A_{\alpha, \beta}^{p}}^{p}}{\left\|f_{\xi, k}\right\|_{A_{\alpha, \beta}^{p}}^{p}}\left\|f_{\xi, k}\right\|_{A_{p(N-1)-2, N-1}^{p}}^{p} \asymp\|u\|_{A_{\alpha, \beta}^{p}}^{p}(k+1)^{2+\alpha-\beta p},
\end{aligned}
$$

therefore $\left(I_{k, 1}\right)_{k}$ is a null sequence in this case. If $\beta \geq \frac{2+\alpha}{p}+1$, then a worse upper bound than the one stated in Lemma 5.4 is given by $C\|u\|_{A_{\alpha, \beta}^{p}}^{p} \frac{1}{\left(1-|z|^{2}\right)^{r}}$ for some positive constant $C$ and any $r>0$. In this case we have, for $0<r<1 / 2$, that

$$
\begin{aligned}
I_{k, 1} & \lesssim\|u\|_{A_{\alpha, \beta}^{p}}^{p} \int_{\mathbb{D}}\left|R^{N-1} g_{\xi, k}\right|^{p}\left(1-|z|^{2}\right)^{-r} d A_{p(N-\beta)+\alpha}(z) \\
& \lesssim \frac{\|u\|_{A_{\alpha, \beta}^{p}}^{p}}{\left\|f_{\xi, k}\right\|_{A_{\alpha, \beta}^{p}}^{p}} \int_{\mathbb{D}}\left|R^{N-1} f_{\xi, k}\right|^{p} d A_{p(N-\beta)+\alpha-r}(z) \\
& =\frac{\|u\|_{A_{\alpha, \beta}^{p}}^{p}}{\left\|f_{\xi, k}\right\|_{A_{\alpha, \beta}^{p}}^{p}}\left\|f_{\xi, k}\right\|_{A_{p(N-\beta)+\alpha-r, N-1}^{p}}^{p} \asymp\|u\|_{A_{\alpha, \beta}^{p}}^{p}(k+1)^{r-p},
\end{aligned}
$$

which completes the proof.

We are now ready to present the main result.

Theorem 4.13. Let $X(\mathbb{D})$ be any of the following spaces:

(a) $\mathcal{B}_{\alpha}(\mathbb{D}), 0<\alpha<1$, with $u \in M\left(\mathcal{B}_{\alpha}(\mathbb{D})\right)=\mathcal{B}_{\alpha}(\mathbb{D}) \subset A(\mathbb{D})$;

(b) $\mathcal{B}(\mathbb{D})$ with $u \in M(\mathcal{B}(\mathbb{D})) \cap A(\mathbb{D})$; 
(c) $A_{\alpha, \beta}^{p}(\mathbb{D})$ with $u \in M\left(A_{\alpha, \beta}^{p}(\mathbb{D})\right)=A_{\alpha, \beta}^{p}(\mathbb{D}) \subset A(\mathbb{D})$, where $p>1$, $\alpha>-1$ and $\beta>\frac{2+\alpha}{p}$;

(d) $H_{\beta}^{2}(\mathbb{D})$ with $u \in M\left(H_{\beta}^{2}(\mathbb{D})\right)=H_{\beta}^{2}(\mathbb{D}) \subset A(\mathbb{D})$, where $\beta>1 / 2$.

Then the essential spectrum of $M_{u}: X(\mathbb{D}) \rightarrow X(\mathbb{D})$ is given by

$$
\sigma_{e}\left(M_{u}\right)=\bigcap_{0<r<1} \overline{u(\mathbb{D} \backslash r \mathbb{D})}=u(\partial \mathbb{D})
$$

Proof. As in the proof of Theorem 4.5, now using Lemmas 4.4, 4.9 and 4.12, we obtain $\sigma_{e}\left(M_{u}\right)=\bigcap_{0<r<1} \overline{u(\mathbb{D} \backslash r \mathbb{D})}$ whenever $u \in M(X(\mathbb{D})) \cap A(\mathbb{D})$ and $X(\mathbb{D})$ is any of the spaces listed above. To prove the last equality, we utilize the continuity of $u$ on $\overline{\mathbb{D}}$, which implies the first equality below

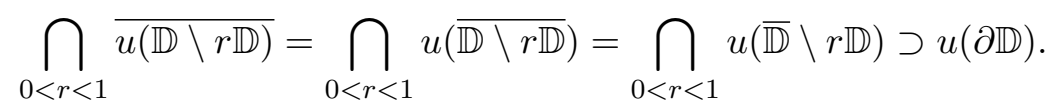

To show the opposite inclusion, take $z \in \bigcap_{0<r<1} u(\overline{\mathbb{D}} \backslash r \mathbb{D})$. Now there is a sequence $\left(y_{n}\right)_{n=1}^{\infty}, 1-\frac{1}{n} \leq\left|y_{n}\right| \leq 1$ such that $u\left(y_{n}\right)=z$. Since $\left(y_{n}\right)_{n=1}^{\infty}$ is bounded there is a convergent subsequence $\left(y_{n_{k}}\right)_{k=1}^{\infty}$ such that $y_{n_{k}} \rightarrow y \in \partial \mathbb{D}$ as $k \rightarrow \infty$. Since $u$ is continuous on $\overline{\mathbb{D}}$ we have

$$
z=\lim _{k \rightarrow \infty} u\left(y_{n_{k}}\right)=u(y),
$$

so $z \in u(\partial \mathbb{D})$, which proves the theorem.

\section{Acknowledgments}

The first two authors were supported in part by the Academy of Finland project 296718 . The third author acknowledges support from the Magnus Ehrnrooth Foundation.

\section{References}

[1] Y. A. Abramovich and C. D. Aliprantis, An Invitation to Operator Theory, Graduate Studies in Mathematics 50, American Mathematical Society, Providence, RI, 2002.

[2] M. Andersson, Topics in Complex Analysis, Universitext, Springer-Verlag, New York, 1997.

[3] S. Axler, Multiplication operators on Bergman spaces, J. Reine Angew. Math. 336 (1982), 26-44.

[4] F. Beatrous and J. Burbea, Holomorphic Sobolev spaces on the ball, Dissertationes Math. (Rozprawy Mat.) 276 (1989), 60 pp. 
[5] _ On multipliers for Hardy-Sobolev spaces, Proc. Amer. Math. Soc. 136 (2008), no. $6,2125-2133$.

[6] K. D. Bierstedt and W. H. Summers, Biduals of weighted Banach spaces of analytic functions, J. Austral. Math. Soc. Ser. A 54 (1993), no. 1, 70-79.

[7] A. Boivin, P. M. Gauthier and C. Zhu, Weighted Hardy spaces for the unit disc: approximation properties, in: Complex and Harmonic Analysis, 129-155, DEStech Publ., Lancaster, PA, 2007.

[8] J. Bonet, P. Domański and M. Lindström, Pointwise multiplication operators on weighted Banach spaces of analytic functions, Studia Math. 137 (1999), no. 2, 176194.

[9] G. Cao and L. He, Fredholmness of multipliers on Hardy-Sobolev spaces, J. Math. Anal. Appl. 418 (2014), no. 1, 1-10.

[10] G. Cao, L. He and K. Zhu, Spectral theory of multiplication operators on HardySobolev spaces, J. Funct. Anal. 275 (2018), no. 5, 1259-1279.

[11] B. R. Choe, H. Koo and W. Smith, Composition operators acting on holomorphic Sobolev spaces, Trans. Amer. Math. Soc. 355 (2003), no. 7, 2829-2855.

[12] M. D. Contreras, J. A. Peláez, C. Pommerenke and J. Rättyä, Integral operators mapping into the space of bounded analytic functions, J. Funct. Anal. 271 (2016), no. 10, 2899-2943.

[13] E. B. Davies, Linear Operators and Their Spectra, Cambridge Studies in Advanced Mathematics 106, Cambridge University Press, Cambridge, 2007.

[14] Q. Fang and J. Xia, Multipliers and essential norm on the Drury-Arveson space, Proc. Amer. Math. Soc. 139 (2011), no. 7, 2497-2504.

[15] W. Koepf, Hypergeometric Summation: An algorithmic approach to summation and special function identities, Advanced Lectures in Mathematics, Friedr. Vieweg \& Sohn, Braunschweig, 1998.

[16] V. Müller, Spectral Theory of Linear Operators and Spectral Systems in Banach Algebras, Operator Theory: Advances and Applications 139, Birkhäuser Verlag, Basel, 2003.

[17] S. Ohno, K. Stroethoff and R. Zhao, Weighted composition operators between Blochtype spaces, Rocky Mountain J. Math. 33 (2003), no. 1, 191-215. 
[18] J. Ortega and J. Fàbrega, Multipliers in Hardy-Sobolev spaces, Integral Equations Operator Theory 55 (2006), no. 4, 535-560.

[19] D. Vukotić, Pointwise multiplication operators between Bergman spaces on simply connected domains, Indiana Univ. Math. J. 48 (1999), no. 3, 793-803.

[20] R. Zhao and K. Zhu, Theory of Bergman spaces in the unit ball of $\mathbb{C}^{n}$, Mém. Soc. Math. Fr. (N.S.) (2008), no. 115, 103 pp.

[21] K. Zhu, Spaces of Holomorphic Functions in the Unit Ball, Graduate Texts in Mathematics 226, Springer-Verlag, New York, 2005.

Mikael Lindström, Santeri Miihkinen and David Norrbo

Department of Mathematics, Åbo Akademi University, FI-20500 Åbo, Finland

E-mail address: mikael.lindstrom@abo.fi, santeri.miihkinen@abo.fi, dnorrbo@abo.fi 